\title{
Carboxylation and Mitsunobu Reaction of Amines to Give Carbamates: Retention vs Inversion of Configuration is Substituent-Dependent
}

\author{
Christopher J. Dinsmore* and Swati P. Mercer \\ Department of Medicinal Chemistry \\ Merck Research Laboratories, West Point, Pennsylvania 19486
}

\begin{abstract}
General Experimental Information: Proton NMR spectra were recorded on Varian Unity or Varian Plus (500 or $400 \mathrm{MHz})$ spectrometers. Chemical shifts are reported in parts per million $(\boldsymbol{\delta})$ downfield from tetramethylsilane as an internal standard. Low resolution electrospray mass spectra were recorded on a Micromass ZMD spectrometer using a Waters 2690 HPLC (2.1 x $100 \mathrm{~mm} \mathrm{C18} \mathrm{column,} \mathrm{H}_{2} \mathrm{O} / \mathrm{MeCN}$ w/0.05\% TFA) for sample introduction. Samples provided for accurate mass measurement were taken up in acetonitrile:water:glacial acetic acid (50:50:0.1\%v/v). The solutions were analyzed by use of electrospray ionization (ESI) or atmospheric pressure chemical ionization (APCI) on either a Bruker Daltonics 3T or 7T Fourier transform ion cyclotron resonance (FTICR) mass spectrometer. External calibration was accomplished with polypropylene glycol (425 or 750). Silica gel 60 (230-400) mesh from EM Science was used for flash column chromatography, and analytical thin layer chromatography was performed on EM Science Kieselgel 60 F254 plates. Solvents and reagents were obtained from commercial sources and used without further purification. The reported yields are the actual isolated yields of purified material and are not optimized.
\end{abstract}

2-[(2-Naphthylmethyl)amino]ethanol (4a): To a solution of ethanolamine $(41.4 \mathrm{~g}, 678 \mathrm{mmol})$ in $600 \mathrm{~mL}$ of acetonitrile at room temperature was added dropwise a solution of 2-(bromomethyl)naphthalene $(10.0 \mathrm{~g}, 45.2 \mathrm{mmol}) \mathrm{in}$ $125 \mathrm{~mL}$ of acetonitrile. After 2 hours, the solution was concentrated in vacuo, and the residue partitioned between dichloromethane and saturated aqueous $\mathrm{NaHCO}_{3}$ solution. The aqueous layer was extracted three times with dichloromethane, and the combined organics were dried $\left(\mathrm{Na}_{2} \mathrm{SO}_{4}\right)$, filtered, and concentrated in vacuo. The crude product was recrystallized from hot EtOAc-hexane to provide $4 \mathbf{a}$ as a white crystalline solid (5.88g, 65\% yield). Analytical LCMS: single peak (1.00 min, $\mathrm{CH}_{3} \mathrm{CN} / \mathrm{H}_{2} \mathrm{O} / 0.05 \%$ TFA, 4 min gradient) $>95 \%$ pure by UV $(215 \mathrm{~nm}) ;{ }^{1} \mathrm{H}$ NMR $\left(500 \mathrm{MHz}, \mathrm{CDCl}_{3}\right) \delta$ 7.79-7.88 (m, 4H), 7.46-7.53 (m, 3H), $4.04(\mathrm{~s}, 2 \mathrm{H}), 3.72(\mathrm{t}, J=5.0 \mathrm{~Hz}, 2 \mathrm{H}), 2.89(\mathrm{t}, J$ $=5.0 \mathrm{~Hz}, 2 \mathrm{H}), 2.16($ br s, $2 \mathrm{H}) ; \mathrm{m} / z\left(\mathrm{ES}^{+}\right)=202.3\left(\mathrm{MH}^{+}\right)$.

General procedure for carboxylation/Mitsunobu cyclization (Table 2):

3-(2-Naphthylmethyl)-1,3-oxazolidin-2-one (6a): To a solution of 2-[(2-naphthylmethyl)amino]ethanol (4a) (100 $\mathrm{mg}, 0.497 \mathrm{mmol}, 1.0$ equiv.) in $2.5 \mathrm{~mL}$ of anhydrous acetonitrile was added 1,8-diazabicyclo[5.4.0]undec-7-ene (7 $\mu \mathrm{L}, 0.05 \mathrm{mmol}, 0.1$ equiv.). The resulting heterogeneous solution was then stirred under an atmosphere of carbon dioxide gas via a balloon, during which the mixture turned homogeneous momentarily and then heterogeneous. After 45 minutes, the reaction was cooled to $0{ }^{\circ} \mathrm{C} . \quad n-\mathrm{Bu}_{3} \mathrm{P}$ was added $(0.260 \mathrm{~mL}, 1.04 \mathrm{mmol}, 2.1$ equiv. $)$, followed by a solution of di-tert-butyl azodicarboxylate $(240 \mathrm{mg}, 1.04 \mathrm{mmol}, 2.1$ equiv.) in $1 \mathrm{ml}$ of acetonitrile in a dropwise manner. Over a period of 20 minutes the system became homogeneous. Removal of the solvent in vacuo afforded a clear yellow residue which was purified by flash chromatography (30-50\% EtOAc/hexanes) to give 1-(2-naphthylmethyl)-2oxazolidinone 6a (104 mg, 92\%) as a white solid. Analytical LCMS: single peak $\left(2.22 \mathrm{~min}, \mathrm{CH}_{3} \mathrm{CN} / \mathrm{H}_{2} \mathrm{O} / 0.05 \% \mathrm{TFA}\right.$, $4 \mathrm{~min}$ gradient) $>97 \%$ pure by UV $(215 \mathrm{~nm}) ;{ }^{1} \mathrm{H}-\mathrm{NMR}\left(500 \mathrm{MHz}, \mathrm{CDCl}_{3}\right) \delta 7.81-7.86(\mathrm{~m}, 3 \mathrm{H}), 7.73(\mathrm{br} \mathrm{s}, 1 \mathrm{H})$, 7.48-7.52 (m, 2H), $7.42(\mathrm{~d}, J=8.5 \mathrm{~Hz}, 1 \mathrm{H}), 4.60(\mathrm{~s}, 2 \mathrm{H}), 4.31(\mathrm{t}, J=7.1 \mathrm{~Hz}, 2 \mathrm{H})$ and $3.45(\mathrm{t}, J=7.1 \mathrm{~Hz}, 2 \mathrm{H}) \mathrm{ppm}$; $m / z\left(\mathrm{ES}^{+}\right)=228.2\left(\mathrm{MH}^{+}\right)$; HRMS (APCI) exact mass calcd for $\mathrm{C}_{14} \mathrm{H}_{14} \mathrm{NO}_{2}\left(\mathrm{M}+\mathrm{H}^{+}\right)$: 228.1019; found 228.1032.

3-Phenyl-1,3-oxazolidin-2-one (6b): ${ }^{1}$ Analytical LCMS: single peak (1.64 min, $\mathrm{CH}_{3} \mathrm{CN} / \mathrm{H}_{2} \mathrm{O} / 0.05 \%$ TFA, $4 \mathrm{~min}$ gradient) $>99 \%$ pure by UV $(215 \mathrm{~nm}) ;{ }^{1} \mathrm{H}$ NMR $\left(500 \mathrm{MHz}, \mathrm{CDCl}_{3}\right) \delta 7.56(\mathrm{br} \mathrm{d}, J=9 \mathrm{~Hz}, 2 \mathrm{H}), 7.39(\mathrm{t}, J=7.5 \mathrm{~Hz}$, $2 \mathrm{H}), 7.15(\mathrm{t}, J=7.5 \mathrm{~Hz}, 1 \mathrm{H}), 4.49(\mathrm{t}, J=8 \mathrm{~Hz}, 2 \mathrm{H}), 4.07(\mathrm{t}, J=8 \mathrm{~Hz}, 2 \mathrm{H}) ; \mathrm{m} / z\left(\mathrm{ES}^{+}\right)=164.2\left(\mathrm{MH}^{+}\right) ; \mathrm{HRMS}^{2}$ (APCI) exact mass calcd for $\mathrm{C}_{9} \mathrm{H}_{10} \mathrm{NO}_{2}\left(\mathrm{M}+\mathrm{H}^{+}\right)$: 164.0706; found 164.0712.

(4R)-4-Benzyl-1,3-oxazolidin-2-one (6c): ${ }^{2}$ Analytical HPLC: single peak (1.73 min, $\mathrm{CH}_{3} \mathrm{CN} / 7.4 \mathrm{mM}$ aq $\mathrm{NH}_{4} \mathrm{OH}, 5$ min gradient) $95.8 \%$ pure by UV $(254 \mathrm{~nm}) ;{ }^{1} \mathrm{H} \mathrm{NMR}\left(500 \mathrm{MHz}, \mathrm{CDCl}_{3}\right) \delta 7.38(\mathrm{t}, J=7.1 \mathrm{~Hz}, 2 \mathrm{H}), 7.30(\mathrm{t}, J=7.1$ $\mathrm{Hz}, 1 \mathrm{H}), 7.20$ (br d, $J=7 \mathrm{~Hz}, 2 \mathrm{H}), 4.95$ (br s, $1 \mathrm{H}), 4.51(\mathrm{dd}, J=9$ and $8 \mathrm{~Hz}, 1 \mathrm{H}), 4.18(\mathrm{dd}, J=8.8 \mathrm{and} 5.6 \mathrm{~Hz}$, 
$1 \mathrm{H}), 4.10(\mathrm{~m}, 1 \mathrm{H}), 2.91(\mathrm{dd}, J=13.4$ and $5.6 \mathrm{~Hz}, 1 \mathrm{H}), 2.86(\mathrm{dd}, J=13.4$ and $8.3 \mathrm{~Hz}, 1 \mathrm{H}) ; \mathrm{m} / z\left(\mathrm{ES}^{+}\right)=178.0$ $\left(\mathrm{MH}^{+}\right)$; HRMS (APCI) exact mass calcd for $\mathrm{C}_{10} \mathrm{H}_{12} \mathrm{NO}_{2}\left(\mathrm{M}+\mathrm{H}^{+}\right)$: 178.0863; found 178.0869.

(4S)-4-Phenyl-1,3-oxazolidin-2-one (6d): ${ }^{3}$ Analytical LCMS: single peak (1.07 min, $\mathrm{CH}_{3} \mathrm{CN} / \mathrm{H}_{2} \mathrm{O} / 0.05 \%$ TFA, 4 min gradient) $97.3 \%$ pure by UV $(215 \mathrm{~nm}) ;{ }^{1} \mathrm{H}$ NMR $\left(500 \mathrm{MHz}, \mathrm{CDCl}_{3}\right) \delta 7.33-7.42(\mathrm{~m}, 5 \mathrm{H}), 5.97(\mathrm{br}, 1 \mathrm{H}), 4.96$ (br t, $J=8 \mathrm{~Hz}, 1 \mathrm{H}), 4.74(\mathrm{t}, J=8.6 \mathrm{~Hz}, 1 \mathrm{H}), 4.19(\mathrm{dd}, J=8.6$ and $6.9 \mathrm{~Hz}, 1 \mathrm{H}) ; \mathrm{m} / z\left(\mathrm{ES}^{+}\right)=164.3\left(\mathrm{MH}^{+}\right) ; \mathrm{HRMS}$ (APCI) exact mass calcd for $\mathrm{C}_{9} \mathrm{H}_{10} \mathrm{NO}_{2}\left(\mathrm{M}+\mathrm{H}^{+}\right)$: 164.0706; found 164.0712.

(5R)-5-Phenyl-1,3-oxazolidin-2-one (6e): ${ }^{4}$ Analytical LCMS: single peak $\left(1.10 \mathrm{~min}, \mathrm{CH}_{3} \mathrm{CN} / \mathrm{H}_{2} \mathrm{O} / 0.05 \%\right.$ TFA, 4 min gradient) $>95 \%$ pure by UV $(215 \mathrm{~nm}) ;{ }^{1} \mathrm{H}$ NMR $\left(500 \mathrm{MHz}, \mathrm{CDCl}_{3}\right) \delta 7.36-7.43(\mathrm{~m}, 5 \mathrm{H}), 5.68(\mathrm{br} \mathrm{s}, 1 \mathrm{H}), 5.63$ (br t, $J=8.2 \mathrm{~Hz}, 1 \mathrm{H}), 3.98(\mathrm{t}, J=8.6 \mathrm{~Hz}, 1 \mathrm{H}), 3.54(\mathrm{t}, J=8 \mathrm{~Hz}, 1 \mathrm{H}) ; \mathrm{m} / z_{2}\left(\mathrm{ES}^{+}\right)=164.2\left(\mathrm{MH}^{+}\right) ; \mathrm{HRMS}(\mathrm{APCI})$ exact mass calcd for $\mathrm{C}_{9} \mathrm{H}_{10} \mathrm{NO}_{2}\left(\mathrm{M}+\mathrm{H}^{+}\right)$: 164.0706; found 164.0709; $[\alpha]_{\mathrm{D}}{ }^{22}=-24.6^{\circ}(c 0.045$, EtOH); literature value for opposite enantiomer: $[\alpha]_{\mathrm{D}}^{25}=+23^{\circ}(c 4.3, \mathrm{EtOH})$.

(4R,5R)-4-(Methoxymethyl)-5-phenyl-1,3-oxazolidin-2-one (6f): ${ }^{5}$ Analytical LCMS: single peak (1.29 min, $\mathrm{CH}_{3} \mathrm{CN} / \mathrm{H}_{2} \mathrm{O} / 0.05 \%$ TFA, 4 min gradient $)>95 \%$ pure by UV $(215 \mathrm{~nm}) ;{ }^{1} \mathrm{H}$ NMR $\left(500 \mathrm{MHz}, \mathrm{CDCl}_{3}\right) \delta 7.35-7.40$ $(\mathrm{m}, 5 \mathrm{H}), 5.77$ (br s, 1H), $5.24(\mathrm{~d}, J=5.6 \mathrm{~Hz}, 1 \mathrm{H}), 3.89$ (br q, $J=6 \mathrm{~Hz}, 1 \mathrm{H}), 3.55(\mathrm{dd}, J=9.5 \mathrm{and} 4.6 \mathrm{~Hz}, 1 \mathrm{H})$, $3.51(\mathrm{dd}, J=9.5$ and $6.9 \mathrm{~Hz}, 1 \mathrm{H}), 3.41(\mathrm{~s}, 3 \mathrm{H}) ; \mathrm{m} / \mathrm{z}\left(\mathrm{ES}^{+}\right)=208.3\left(\mathrm{MH}^{+}\right)$; HRMS (APCI) exact mass calcd for $\mathrm{C}_{10} \mathrm{H}_{14} \mathrm{NO}\left(\mathrm{M}+\mathrm{H}^{+}-\mathrm{CO}_{2}\right): 164.1075$; found 164.1075 . Proof of structure was provided by hydrolysis to give $(1 R, 2 R)-$ 2-amino-3-methoxy-1-phenylpropan-1-ol (4f). To a solution of $\mathbf{6 f}(87 \mathrm{mg}, 0.42 \mathrm{mmol})$ in $1 \mathrm{~mL}$ of ethanol was added $0.5 \mathrm{~mL}$ of $20 \%$ aqueous $\mathrm{KOH}$ solution. The mixture was heated at $60{ }^{\circ} \mathrm{C}$ for 3 days, then cooled to room temperature. The solution was partitioned between $\mathrm{CHCl}_{3}$ and water, and the aqueous layer was extracted three times with $\mathrm{CHCl}_{3}$. The combined organic layers were dried $\left(\mathrm{Na}_{2} \mathrm{SO}_{4}\right)$, filtered, and concentrated in vacuo. Purification by flash column chromatography (7-10\% $\mathrm{MeOH} / \mathrm{CH}_{2} \mathrm{Cl}_{2}$ ) gave $4 \mathbf{f}$ as a colorless oil (38.8 $\mathrm{mg}$, $50 \%$ yield), which was spectroscopically identical ( ${ }^{\mathrm{H}} \mathrm{NMR}$, Analytical LCMS) to commercial material, and had the correct sign of optical rotation: $[\alpha]_{\mathrm{D}}^{22}=+19.2^{\circ}\left(c 0.030, \mathrm{CHCl}_{3}\right)$; literature value: $[\alpha]_{\mathrm{D}}^{25}=+25^{\circ}\left(c 10.6, \mathrm{CHCl}_{3}\right)$.

(4R,5S)-4-Methyl-5-phenyl-1,3-oxazolidin-2-one (6g): ${ }^{6}$ Analytical HPLC: single peak (1.92 min, $\mathrm{CH}_{3} \mathrm{CN} / 7.4 \mathrm{mM}$ aq $\mathrm{NH}_{4} \mathrm{OH}, 5 \mathrm{~min}$ gradient) $>99 \%$ pure by UV $(254 \mathrm{~nm}) ;{ }^{1} \mathrm{H} \mathrm{NMR}\left(500 \mathrm{MHz}, \mathrm{CDCl}_{3}\right) \delta 7.36-7.43(\mathrm{~m}, 3 \mathrm{H}), 7.32$ (br d, $J=8 \mathrm{~Hz}, 1 \mathrm{H}), 5.74(\mathrm{~d}, J=7.8 \mathrm{~Hz}, 1 \mathrm{H}), 5.08(\mathrm{br} \mathrm{s}, 1 \mathrm{H}), 4.22(\mathrm{dq}, J=7$ and $7 \mathrm{~Hz}, 1 \mathrm{H}), 0.83(\mathrm{~d}, J=7.8 \mathrm{~Hz}$, $3 \mathrm{H}) ; \mathrm{m} / z\left(\mathrm{ES}^{+}\right)=178.2\left(\mathrm{MH}^{+}\right)$; HRMS (APCI) exact mass calcd for $\mathrm{C}_{10} \mathrm{H}_{12} \mathrm{NO}_{2}\left(\mathrm{M}+\mathrm{H}^{+}\right)$: 178.0863; found 178.0870 .

(4R,5R)-3,4-Dimethyl-5-phenyl-1,3-oxazolidin-2-one (6h): ${ }^{7}$ Analytical HPLC: single peak (2.14 min, $\mathrm{CH}_{3} \mathrm{CN} / 7.4$ $\mathrm{mM}$ aq $\mathrm{NH}_{4} \mathrm{OH}, 5 \mathrm{~min}$ gradient $)>99 \%$ pure by UV $(254 \mathrm{~nm}) ;{ }^{1} \mathrm{H} \mathrm{NMR}\left(500 \mathrm{MHz}, \mathrm{CDCl}_{3}\right) \delta 7.37-7.43(\mathrm{~m}, 5 \mathrm{H})$, $4.92(\mathrm{~d}, J=7.8 \mathrm{~Hz}, 1 \mathrm{H}), 3.55(\mathrm{dq}, J=7$ and $7 \mathrm{~Hz}, 1 \mathrm{H}), 2.88(\mathrm{~s}, 3 \mathrm{H}), 1.38(\mathrm{~d}, J=7.8 \mathrm{~Hz}, 3 \mathrm{H}) ; m / z\left(\mathrm{ES}^{+}\right)=192.0$ $\left(\mathrm{MH}^{+}\right)$; HRMS (APCI) exact mass calcd for $\mathrm{G}_{1} \mathrm{H}_{14} \mathrm{NO}_{2}\left(\mathrm{M}+\mathrm{H}^{+}\right): 192.1019$; found 192.1024; $[\alpha]_{\mathrm{D}}^{22}=-30^{\circ}(c$ $\left.0.040, \mathrm{CHCl}_{3}\right)$.

(4R,5S)-4,5-Diphenyl-1,3-oxazolidin-2-one (6i): ${ }^{8}$ Analytical LCMS: sngle peak $\left(1.73 \mathrm{~min}, \mathrm{CH}_{3} \mathrm{CN} / \mathrm{H}_{2} \mathrm{O} / 0.05 \%\right.$ TFA, 4 min gradient) $>97 \%$ pure by UV $(215 \mathrm{~nm}) ;{ }^{1} \mathrm{H}$ NMR $\left(400 \mathrm{MHz}, \mathrm{CDCl}_{3}\right) \delta 7.01-7.07(\mathrm{~m}, 6 \mathrm{H}), 6.87-6.92(\mathrm{~m}$, $4 \mathrm{H}), 5.89(\mathrm{~d}, J=8.3 \mathrm{~Hz}, 1 \mathrm{H}), 5.73(\mathrm{br} \mathrm{s}, 1 \mathrm{H}), 5.13(\mathrm{~d}, J=8.3 \mathrm{~Hz}, 1 \mathrm{H}) ; \mathrm{m} / z\left(\mathrm{ES}^{+}\right)=240.3\left(\mathrm{MH}^{+}\right) ; \mathrm{HRMS}(\mathrm{APCI})$ exact mass calcd for $\mathrm{C}_{15} \mathrm{H}_{14} \mathrm{NO}_{2}\left(\mathrm{M}+\mathrm{H}^{+}\right): 240.1019$; found $240.1025 ;[\alpha]_{\mathrm{D}}^{22}=+80.8^{\circ}\left(c 0.013, \mathrm{CHCl}_{3}\right)(\mathrm{measured}$ $[\alpha]_{\mathrm{D}}^{22}=-80.4^{\circ}\left(c 0.013, \mathrm{CHCl}_{3}\right)$ for authentic commercially available opposite enantiomer $)$.

(4S,5S)-3-Benzyl-4,5-diphenyl-1,3-oxazolidin-2-one (6j): Analytical LCMS: single peak (3.20 min, $\mathrm{CH}_{3} \mathrm{CN} / \mathrm{H}_{2} \mathrm{O} / 0.05 \%$ TFA, 4 min gradient) $94 \%$ pure by UV $(215 \mathrm{~nm}) ;{ }^{1} \mathrm{H}$ NMR $(500 \mathrm{MHz}, \mathrm{CDCl}) \delta 7.38-7.41(\mathrm{~m}$, $3 \mathrm{H}), 7.26-7.29(\mathrm{~m}, 3 \mathrm{H}), 7.22-7.24(\mathrm{~m}, 6 \mathrm{H}), 7.17(\mathrm{~m}, 1 \mathrm{H}), 7.10(\mathrm{~m}, 1 \mathrm{H}), 7.07(\mathrm{~m}, 1 \mathrm{H}), 5.22(\mathrm{~d}, J=7.4 \mathrm{~Hz}, 1 \mathrm{H})$, $4.90(\mathrm{~d}, J=14.9 \mathrm{~Hz}, 1 \mathrm{H}), 4.25(\mathrm{~d}, J=7.4 \mathrm{~Hz}, 1 \mathrm{H}), 3.67(\mathrm{~d}, J=14.9 \mathrm{~Hz}, 1 \mathrm{H}) ; \mathrm{m} / z\left(\mathrm{ES}^{+}\right)=330.1\left(\mathrm{MH}^{+}\right) ; \mathrm{HRMS}$ (APCI) exact mass calcd for $\mathrm{C}_{22} \mathrm{H}_{20} \mathrm{NO}_{2}\left(\mathrm{M}+\mathrm{H}^{+}\right)$: 330.1489; found 330.1479. 

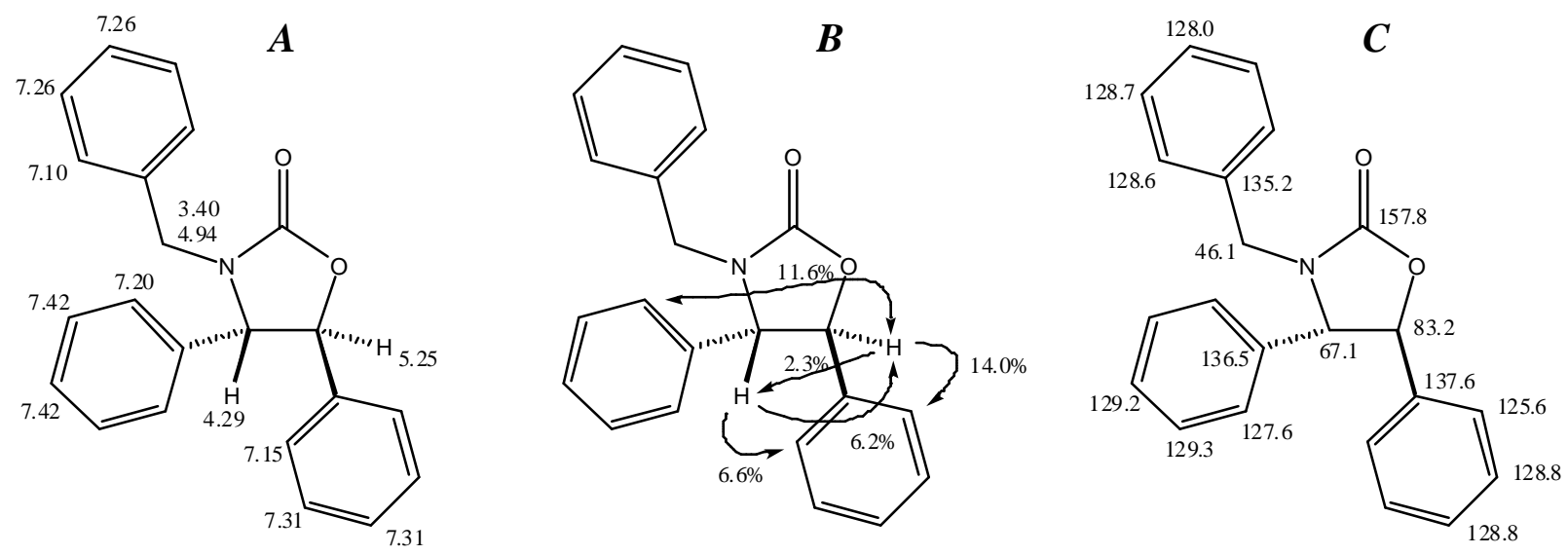

Figure 1. NMR spectral assignments for $\mathbf{6 j}$ derived from ${ }^{1} \mathrm{H}$ NMR $\left(600 \mathrm{MHz}, \mathrm{CDCl}_{3}\right)$, $\mathrm{HMQC}, \mathrm{qHMBC}$ and difference NOE experiments. $A:{ }^{1} \mathrm{H}$ NMR assignments (ppm); B: NOE signal enhancements (\%); $C:{ }^{13} \mathrm{C}$ NMR assignments (ppm).

Support for the structure assignment was provided by hydrolysis to give (1S,2S)-2-(benzylamino)-1,2-diphenylethanol. To a solution of $\mathbf{6 j}$ ( $20 \mathrm{mg}, 0.061 \mathrm{mmol}$ ) in $1 \mathrm{~mL}$ of ethanol was added $0.5 \mathrm{~mL}$ of $20 \%$ aqueous $\mathrm{KOH}$ solution. The mixture was heated to $60^{\circ} \mathrm{C}$ for 24 hours, after which another portion of $20 \%$ aqueous $\mathrm{KOH}$ solution was added. After 1 hour the solution was cooled, then extracted three times with $\mathrm{CH}_{2} \mathrm{Cl}_{2}$. The combined organic layers were dried $\left(\mathrm{Na}_{2} \mathrm{SO}_{4}\right)$, filtered, and concentrated in vacuo to give an oil $\left(\mathrm{m} / z\left(\mathrm{ES}^{+}\right)=304.2\left(\mathrm{MH}^{+}\right)\right)$, the ${ }^{-1} \mathrm{H}$ NMR spectrum of which does not match commercially available (1R,2S)-2-(benzylamino)-1,2-diphenylethanol (4j).

(3aR,7aS)-3-Benzylhexahydro-1,3-benzoxazol-2(3H)-one (6k): ${ }^{9}$ Analytical LCMS: single peak (2.31 min, $\mathrm{CH}_{3} \mathrm{CN} / \mathrm{H}_{2} \mathrm{O} / 0.05 \% \mathrm{TFA}, 4 \mathrm{~min}$ gradient) $>97 \%$ pure by UV $(215 \mathrm{~nm}) ;{ }^{1} \mathrm{H}$ NMR $\left(500 \mathrm{MHz}, \mathrm{CDCl}_{3}\right) \delta 7.30-7.38$ $(\mathrm{m}, 5 \mathrm{H}), 4.80(\mathrm{~d}, J=15.1 \mathrm{~Hz}, 1 \mathrm{H}), 4.48(\mathrm{q}, J=5 \mathrm{~Hz}, 1 \mathrm{H}), 4.06(\mathrm{~d}, J=15.1 \mathrm{~Hz}, 1 \mathrm{H}), 3.51(\mathrm{q}, J=7 \mathrm{~Hz}, 1 \mathrm{H}), 1.95$ (m, 1H), $1.77(\mathrm{~m}, 2 \mathrm{H}), 1.58(\mathrm{~m}, 2 \mathrm{H}), 1.50(\mathrm{~m}, 1 \mathrm{H}), 1.44(\mathrm{~m}, 1 \mathrm{H}), 1.24(\mathrm{~m}, 1 \mathrm{H}) ; \mathrm{m} / z\left(\mathrm{ES}^{+}\right)=232.1\left(\mathrm{MH}^{+}\right) ; \mathrm{HRMS}$ (APCI) exact mass calcd for $\mathrm{C}_{14} \mathrm{H}_{18} \mathrm{NO}_{2}\left(\mathrm{M}+\mathrm{H}^{+}\right)$: 232.1332; found 232.1341. Support for the structure assignment was provided by hydrolysis to give $(1 S, 2 R)$-2-(benzylamino)cyclohexanol. To a solution of $\mathbf{6 k}(20 \mathrm{mg}, 0.086 \mathrm{mmol})$ in $1 \mathrm{~mL}$ of ethanol was added $0.5 \mathrm{~mL}$ of $20 \%$ aqueous $\mathrm{KOH}$ solution. The mixture was heated to $60^{\circ} \mathrm{C}$ for 24 hours, after which another portion of $20 \%$ aqueous $\mathrm{KOH}$ solution was added. After 48 hours, the solution was cooled, then extracted three times with $\mathrm{CH}_{2} \mathrm{Cl}$. The combined organic layers were dried $\left(\mathrm{Na}_{2} \mathrm{SO}_{4}\right)$, filtered, and concentrated in vacuo to give an oil $\left(\mathrm{m} / z\left(\mathrm{ES}^{+}\right)=206.3\left(\mathrm{MH}^{+}\right)\right)$, the ${ }^{1} \mathrm{H}$ NMR spectrum of which does not match commercially available $(1 R, 2 R)$-2-(benzylamino)cyclohexanol $(\mathbf{4 k})$.
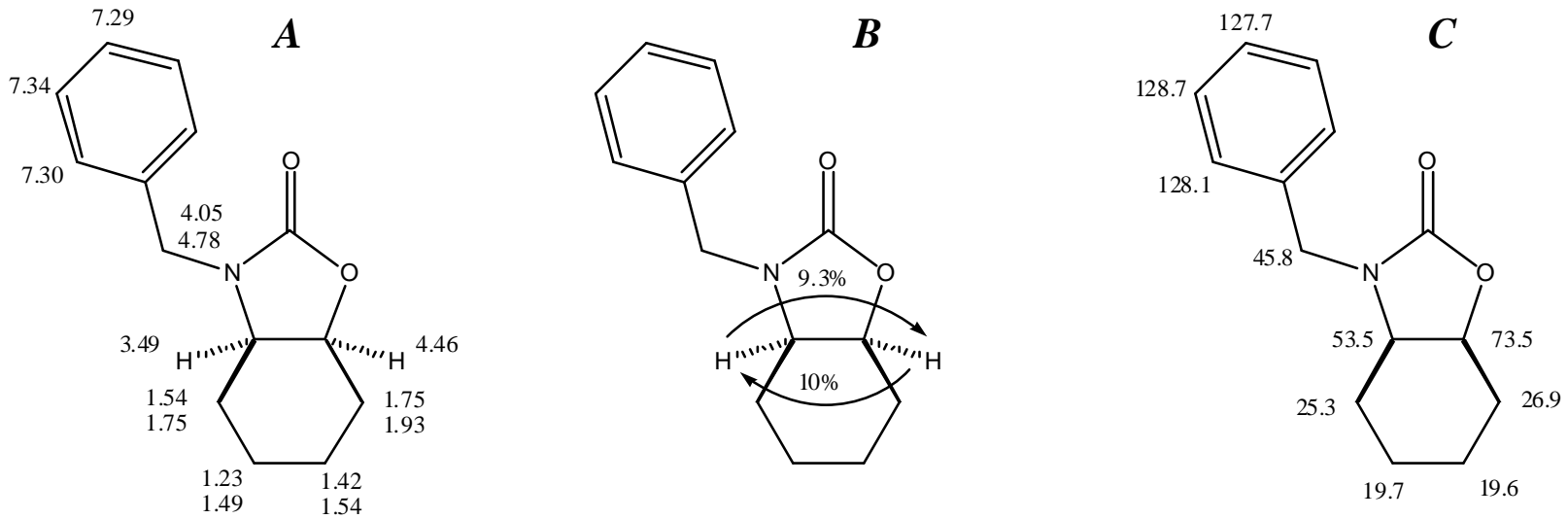

Figure 2. NMR spectral assignments for $\mathbf{6 k}$ derived from ${ }^{1} \mathrm{H}$ NMR $\left(600 \mathrm{MHz}, \mathrm{CDCl}_{3}\right)$, $\mathrm{HMQC}, \mathrm{GCOSY}$ and difference NOE experiments. $A:{ }^{1} \mathrm{H}$ NMR assignments (ppm); $B: \mathrm{NOE}$ signal enhancements (\%); $C:{ }^{13} \mathrm{C}$ NMR assignments (ppm).

( \pm )-4-Phenyl-1,3-oxazinan-2-one (9): ${ }^{10}$ Analytical LCMS: single peak (1.04 min, $\mathrm{CH}_{3} \mathrm{CN} / \mathrm{H}_{2} \mathrm{O} / 0.05 \%$ TFA, 4 min gradient) >97\% pure by UV (215 nm); ${ }^{1} \mathrm{H}$ NMR $\left(500 \mathrm{MHz}, \mathrm{CDCl}_{3}\right) \delta 7.39$ (br t, $\left.J=8 \mathrm{~Hz}, 2 \mathrm{H}\right), 7.31-7.35(\mathrm{~m}, 3 \mathrm{H})$, 
$5.55($ br s, $1 \mathrm{H}), 4.66(\mathrm{t}, J=6.1 \mathrm{~Hz}, 1 \mathrm{H}), 4.28-4.34(\mathrm{~m}, 2 \mathrm{H}), 2.26(\mathrm{~m}, 1 \mathrm{H}), 1.98(\mathrm{~m}, 1 \mathrm{H}) ; m / z\left(\mathrm{ES}^{+}\right)=178.3\left(\mathrm{MH}^{+}\right)$; HRMS (APCI) exact mass calcd for $\mathrm{C}_{10} \mathrm{H}_{12} \mathrm{NO}_{2}\left(\mathrm{M}+\mathrm{H}^{+}\right)$: 178.0863 ; found 178.0870.

\section{Intermolecular carboxylation/Mitsunobu reaction (eq 4):}

Benzyl 3,4-dihydroisoquinoline-2(1H)-carboxylate (23): ${ }^{11}$ To a solution of 1,2,3,4-tetrahydroisoquinoline (22) (203 mg, 0.1.52 mmol, 1.0 equiv.) and benzyl alcohol $(0.237 \mathrm{~mL}, 2.29 \mathrm{mmol}, 1.5$ equiv.) in $7 \mathrm{~mL}$ of anhydrous acetonitrile was added 1,8-diazabicyclo[5.4.0]undec-7-ene $(23 \mu \mathrm{L}, 0.15 \mathrm{mmol}, 0.1$ equiv.). The resulting solution was then stirred under an atmosphere of carbon dioxide gas via a balloon, during which the mixture remained homogeneous. After 45 minutes, the reaction was cooled to $0{ }^{\circ} \mathrm{C} .{ }^{n}$ - $\mathrm{Bu}_{3} \mathrm{P}$ was added $(0.799 \mathrm{~mL}, 3.20 \mathrm{mmol}, 2.1$ equiv. $)$, followed by a solution of di-tert-butyl azodicarboxylate $(737 \mathrm{mg}, 3.20 \mathrm{mmol}, 2.1$ equiv.) in $2 \mathrm{ml}$ of acetonitrile in a dropwise manner. After 20 minutes, removal of the solvent in vacuo afforded a clear yellow residue which was purified by flash chromatography (50-80\% $\mathrm{CH}_{2} \mathrm{Cl}_{2}$ /hexanes) to give benzyl 3,4-dihydroisoquinoline-2(1H)-carboxylate 23 (365 mg, 90\%) as a colorless oil. Analytical LCMS: single peak (2.49 min, $\mathrm{CH}_{3} \mathrm{CN} / \mathrm{H}_{2} \mathrm{O} / 0.05 \%$ TFA, 4 min gradient) $97 \%$ pure by UV $(215 \mathrm{~nm}) ;{ }^{1} \mathrm{H}-\mathrm{NMR}\left(500 \mathrm{MHz}, \mathrm{CDCl}_{3}\right) \delta$ 7.33-7.42 (m, 4H), 7.14-7.21 (m, 5H), $5.20(\mathrm{~s}, 2 \mathrm{H}), 4.67(\mathrm{~s}, 2 \mathrm{H})$, 3.75 (br t, $\mathrm{J}=5 \mathrm{~Hz}, 2 \mathrm{H})$ and $2.87($ br s, $2 \mathrm{H}) \mathrm{ppm} ; \mathrm{m} / \mathrm{z}\left(\mathrm{ES}^{+}\right)=268.2\left(\mathrm{MH}^{+}\right) ; \mathrm{m} / z(\mathrm{EI})=176\left(\mathrm{C}_{10} \mathrm{H}_{10} \mathrm{NO}_{2}\right), 132$ $\left(\mathrm{C}_{9} \mathrm{H}_{10} \mathrm{~N}\right), 91\left(\mathrm{C}_{7} \mathrm{H}_{7}\right)$.

Procedure for carboxylation/Mitsunobu cyclization using ${ }^{18} \mathrm{O}$-labeled $\mathrm{CO}_{2}$ (eq 2 and 3):

(Compounds 10 and 11): A mixture of aminoalcohol (either $\mathbf{4 g}$ or $\mathbf{4 h}, 0.33 \mathrm{mmol}, 1.0$ equiv.) and 1,8diazabicyclo[5.4.0]undec-7-ene (5 uL, $0.03 \mathrm{mmol}, 0.1$ equiv.) in $3 \mathrm{~mL}$ of anhydrous acetonitrile was degassed by bubbling argon through the solution for 20 minutes. The resulting solution was then stirred under an atmosphere of ${ }^{18} \mathrm{O}$ labeled carbon dioxide gas (97 atom\% ${ }^{18} \mathrm{O}$; ISOTEC lot no. CR0916) via balloon, during which the mixture remained homogeneous. After 45 minutes, the reaction was cooled to $0{ }^{\circ} \mathrm{C} . n$ - $\mathrm{Bu}_{3} \mathrm{P}$ was added $(0.173 \mathrm{~mL}, 0.69 \mathrm{mmol}, 2.1$ equiv.), followed by a solution of di-tert-butyl azodicarboxylate (160 mg, $0.69 \mathrm{mmol}, 2.1 \mathrm{equiv}$.) in $0.5 \mathrm{ml}$ of degassed acetonitrile in a dropwise manner. After 20 minutes, LCMS analysis indicated the presence of a single product peak. Removal of the solvent in vacuo afforded a clear yellow residue which was analyzed directly by LCMS (ES), HRMS (APCI) and ${ }^{1} \mathrm{H}$ NMR spectroscopy. The ${ }^{1} \mathrm{H}$ NMR $(500 \mathrm{MHz})$ spectrum of the crude reaction mixture containing 10 was identical to that for crude $\mathbf{6 g}$, and the corresponding spectrum of the crude reaction mixture containing $\mathbf{1 1}$ was identical to that for crude $\mathbf{6 h}$. Copies of the LCMS and HRMS spectra from these experiments are shown at the end of this document.

\section{References:}

(1) Cacchi, S.; Fabrizi, G.; Goggiamani, A.; Zappia, G. Org. Lett. 2001, 3, 2539.

(2) Tadao, I.; Koreichi, K.; Seigo, I.; Takehisa, K. Chem. Lett. 1992, 991.

(3) Schoepf, C.; Wuest, W. Justus Liebigs Ann. Chem. 1959, 626, 150.

(4) Benedetti, F.; Norbedo, S. Tetrahedron Lett. 2000, 41, 10071.

(5) Meyers, A. I.; Ford, M. E. J. Org. Chem. 1976, 41, 1735.

(6) Kano, S.; Yokomatsu, T.; Iwasawa, H.; Shibuya, S. Tetrahedron Lett. 1987, 28, 6331.

(7) Fodor, G.; Stefanovsky, J.; Kurtev, B. Monatsh. Chem. 1967, 98, 1027.

(8) Park, Y. S.; Boys, M. L.; Beak, P. J. Am. Chem. Soc. 1996, 118, 3757.

(9) Van Delft, F. L.; Timmers, C. M.; Van der Marel, G. A.; Van Boom, J. H.. Synthesis. 1997, 450.

(10) Lagu, B.; Dhar, T. M.; Nagarathnam, D.; Jeon, Y. T.; Marzabadi, M. R.; Wong, W. C.; Gluchowski, C. US 6,159,990, 2000.

(11) Ihara, M.; Hirabayashi, A.; Taniguchi, N.; Fukumoto, K. Heterocycles 1992, 33, 851.

\section{Following Pages:}

Copies of the ${ }^{1} \mathrm{H}$ NMR spectra for compounds 6a-k, 9 and 23: 


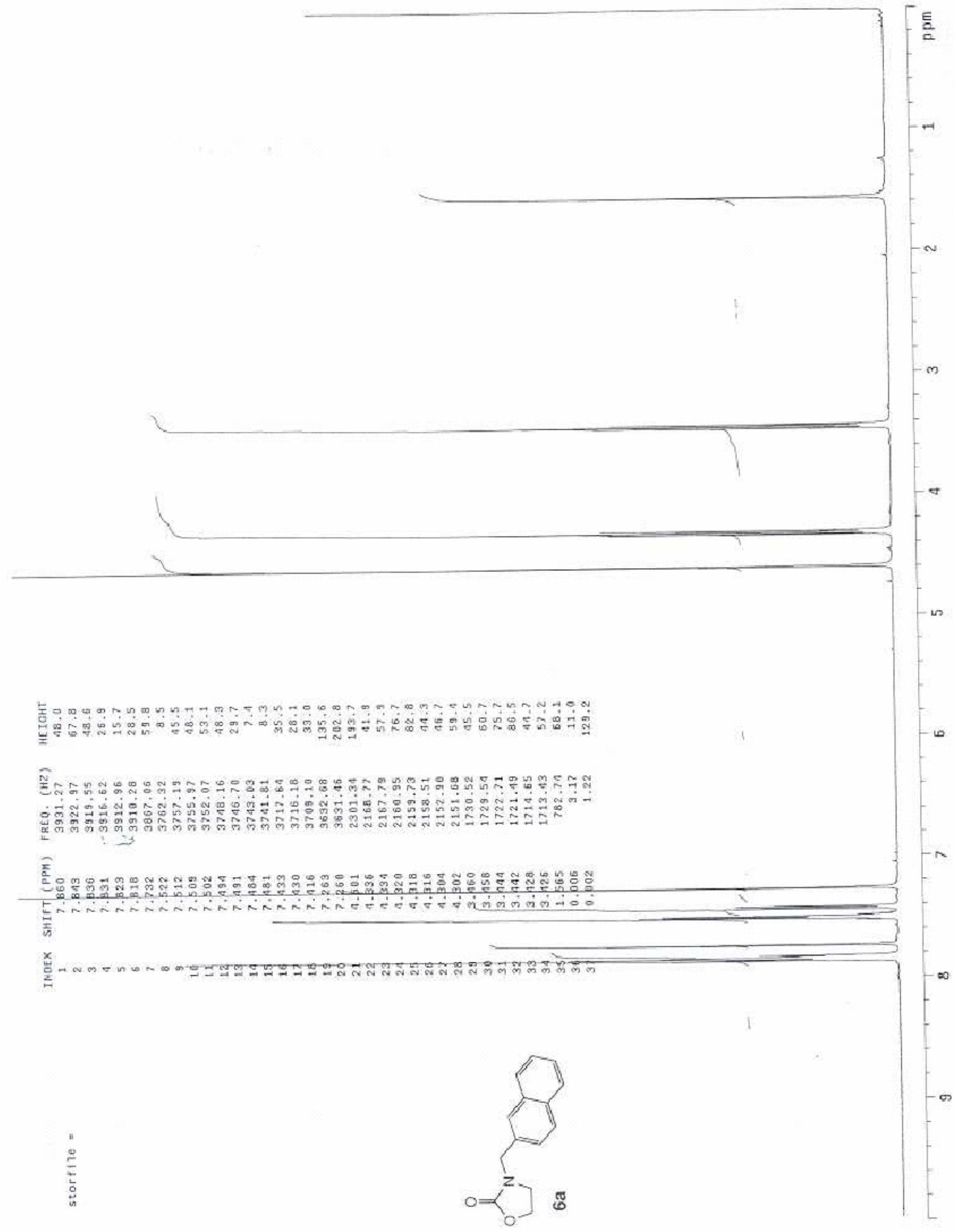




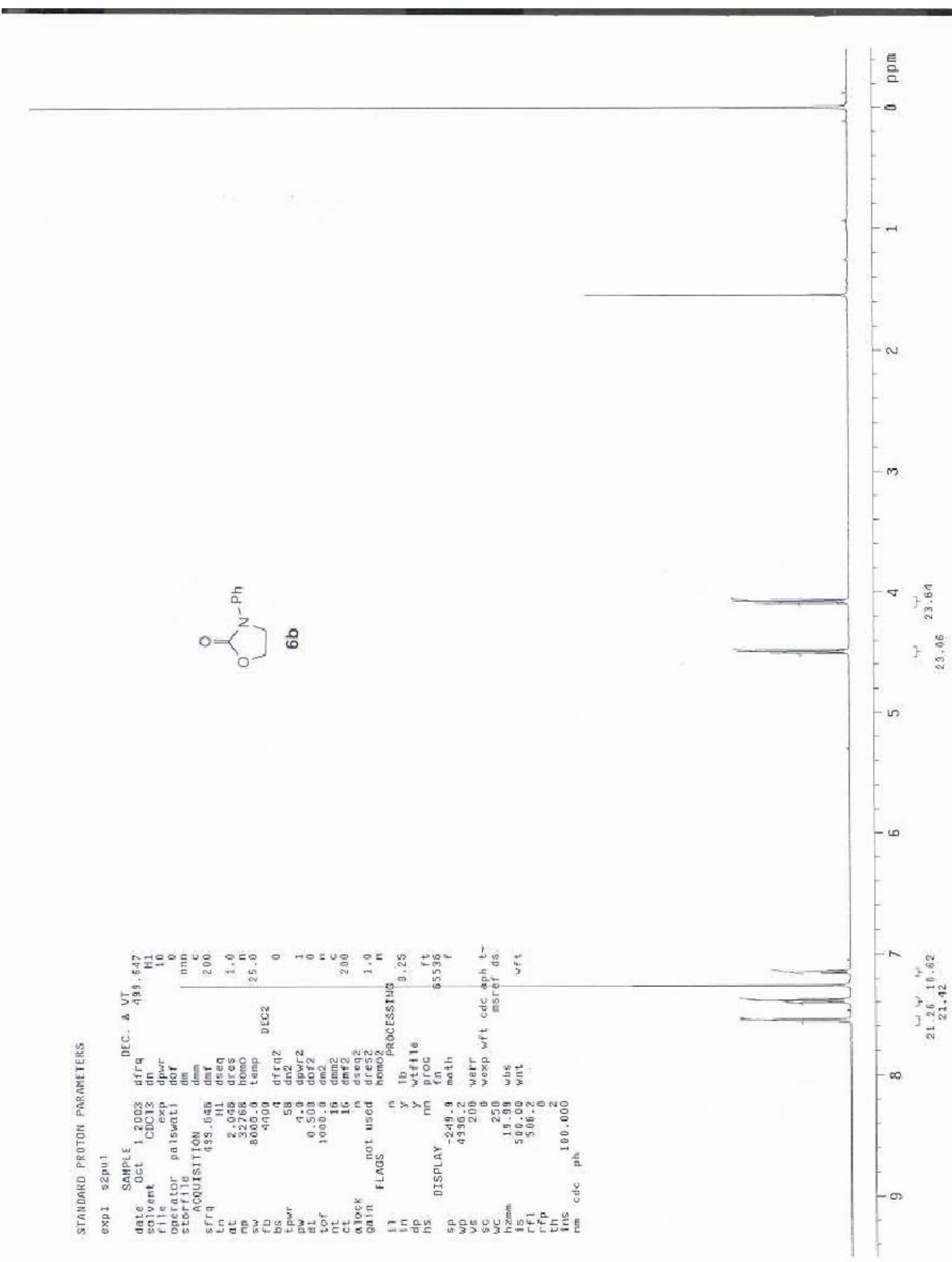




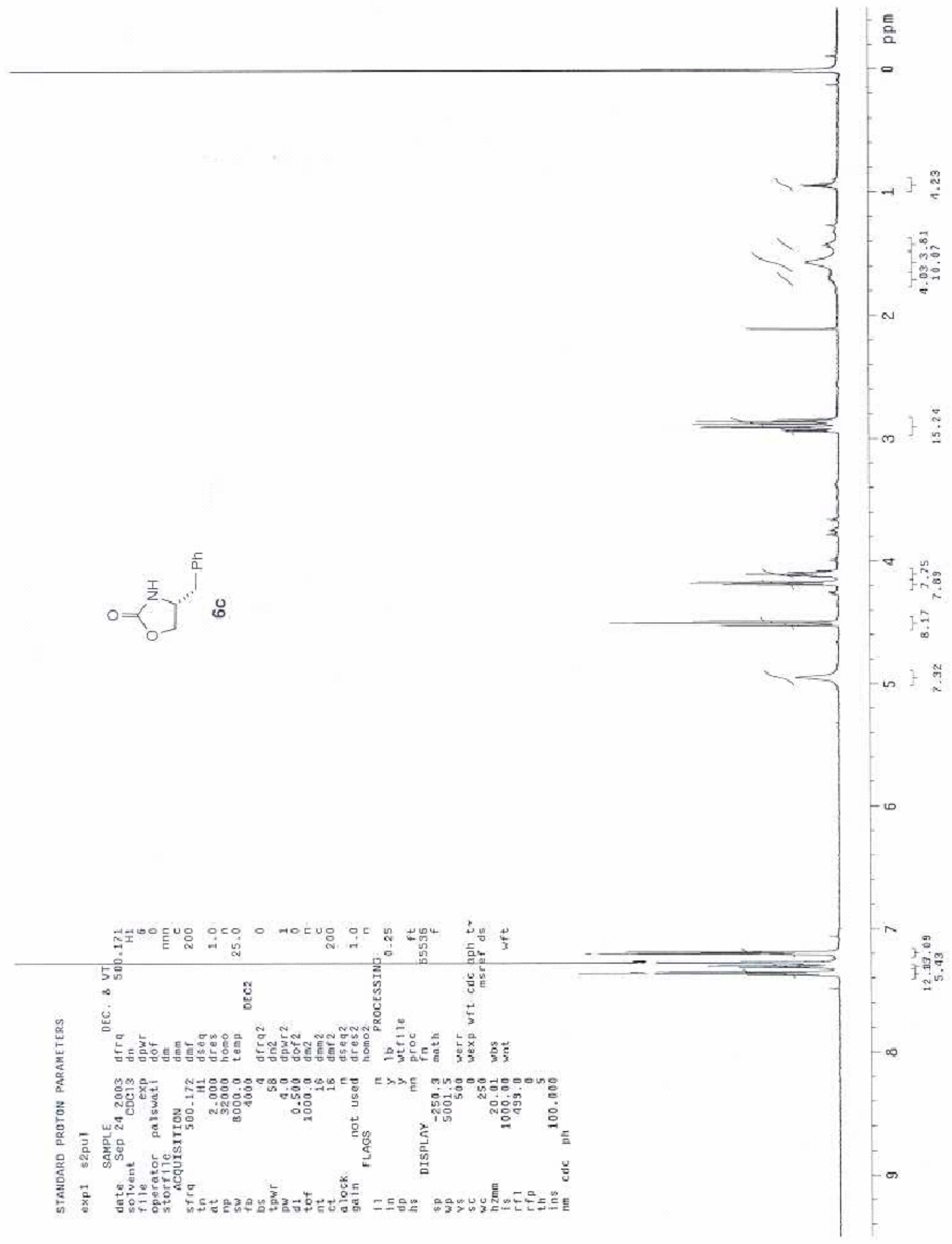




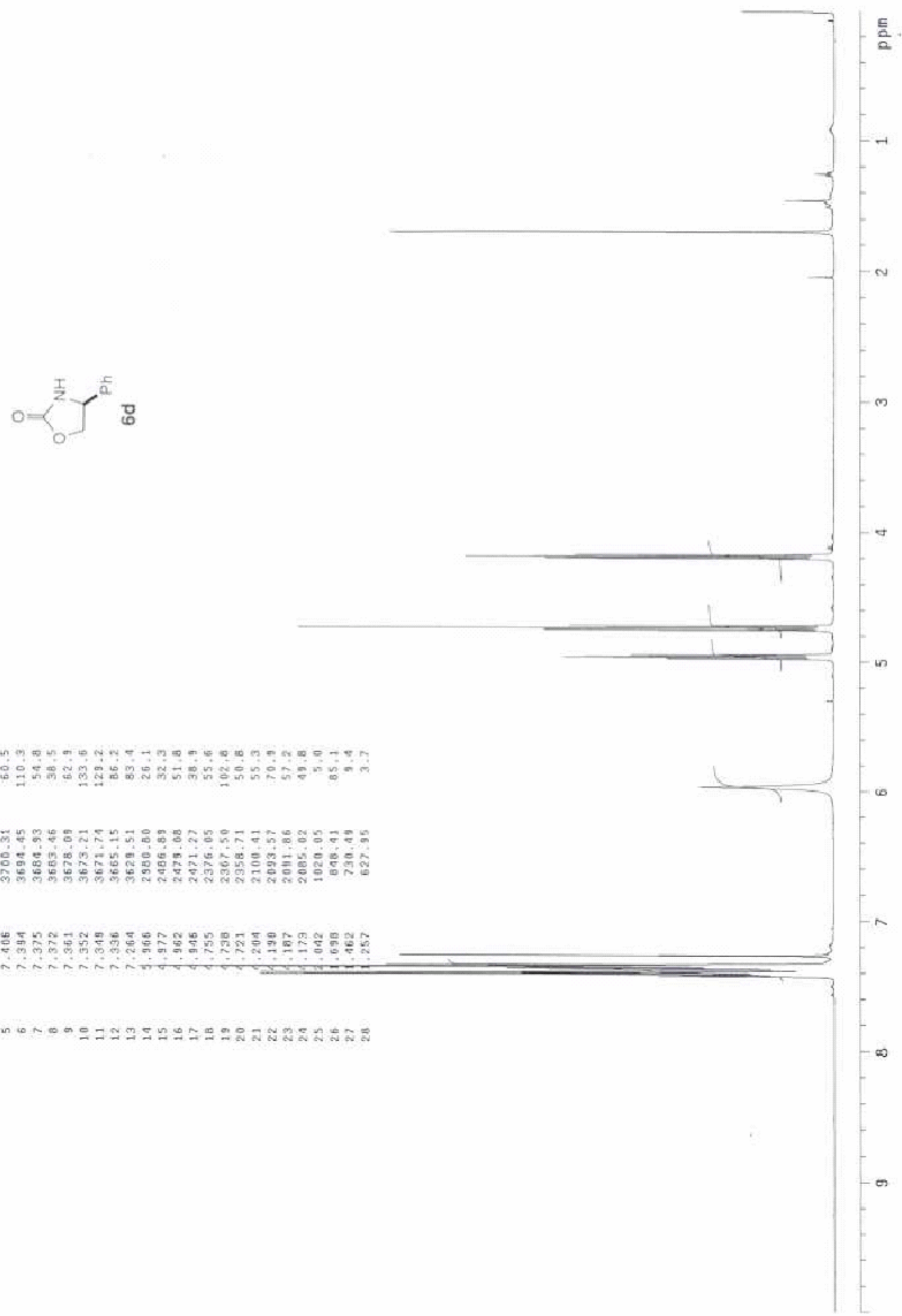

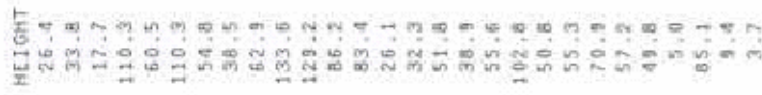

药

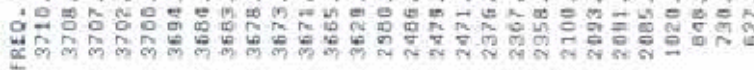

高

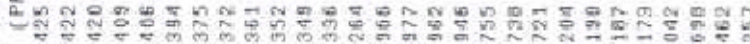
常

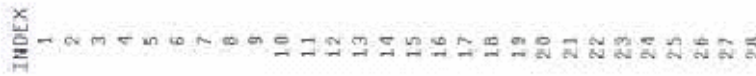

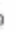

$$
\frac{5}{\square}
$$




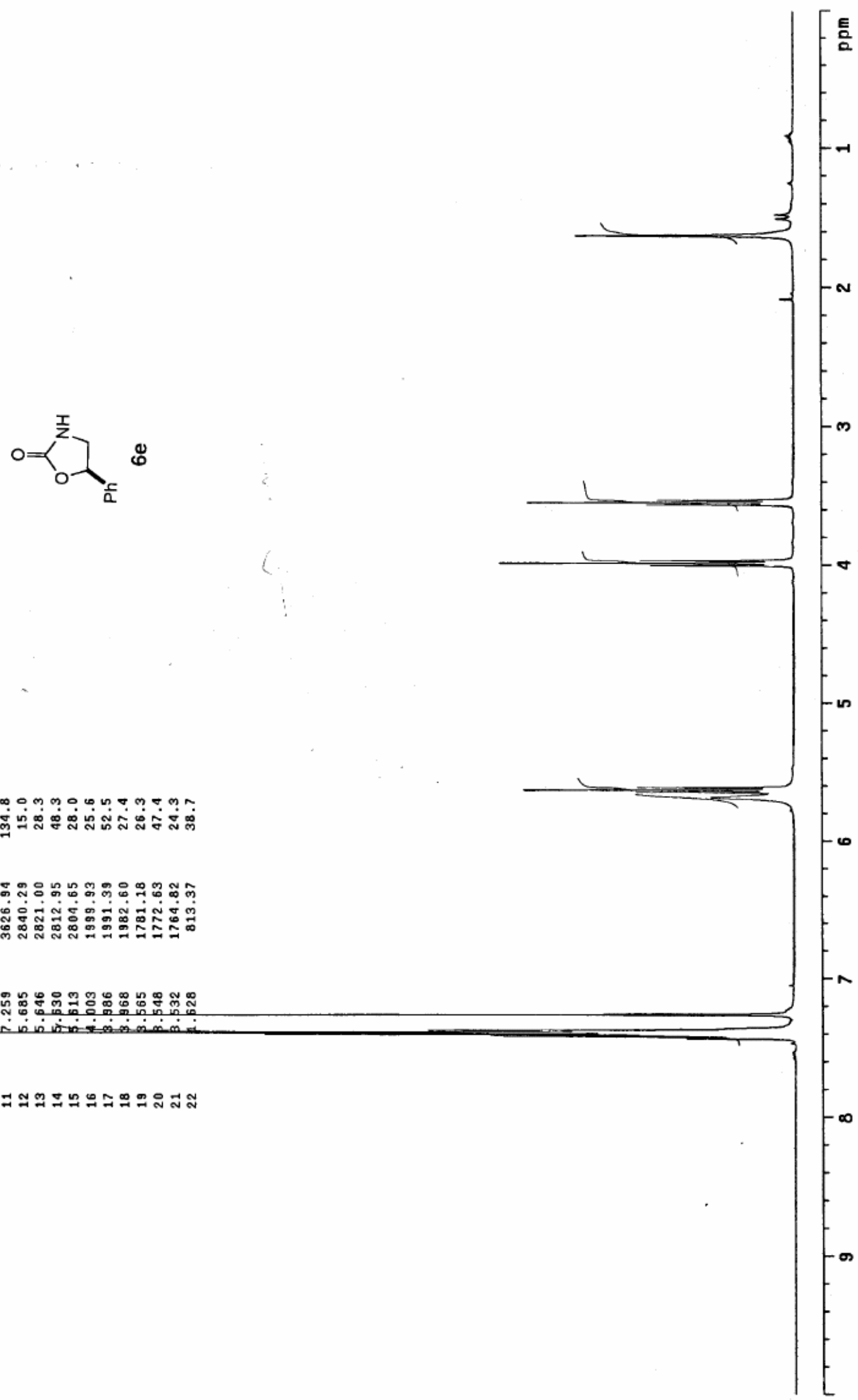

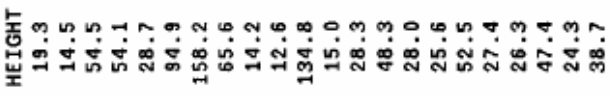

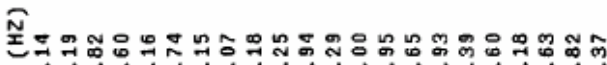

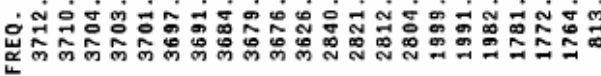

产.

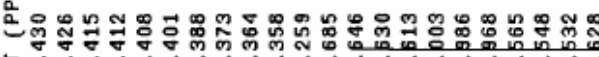

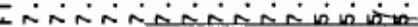

辛

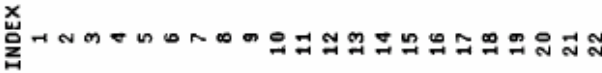




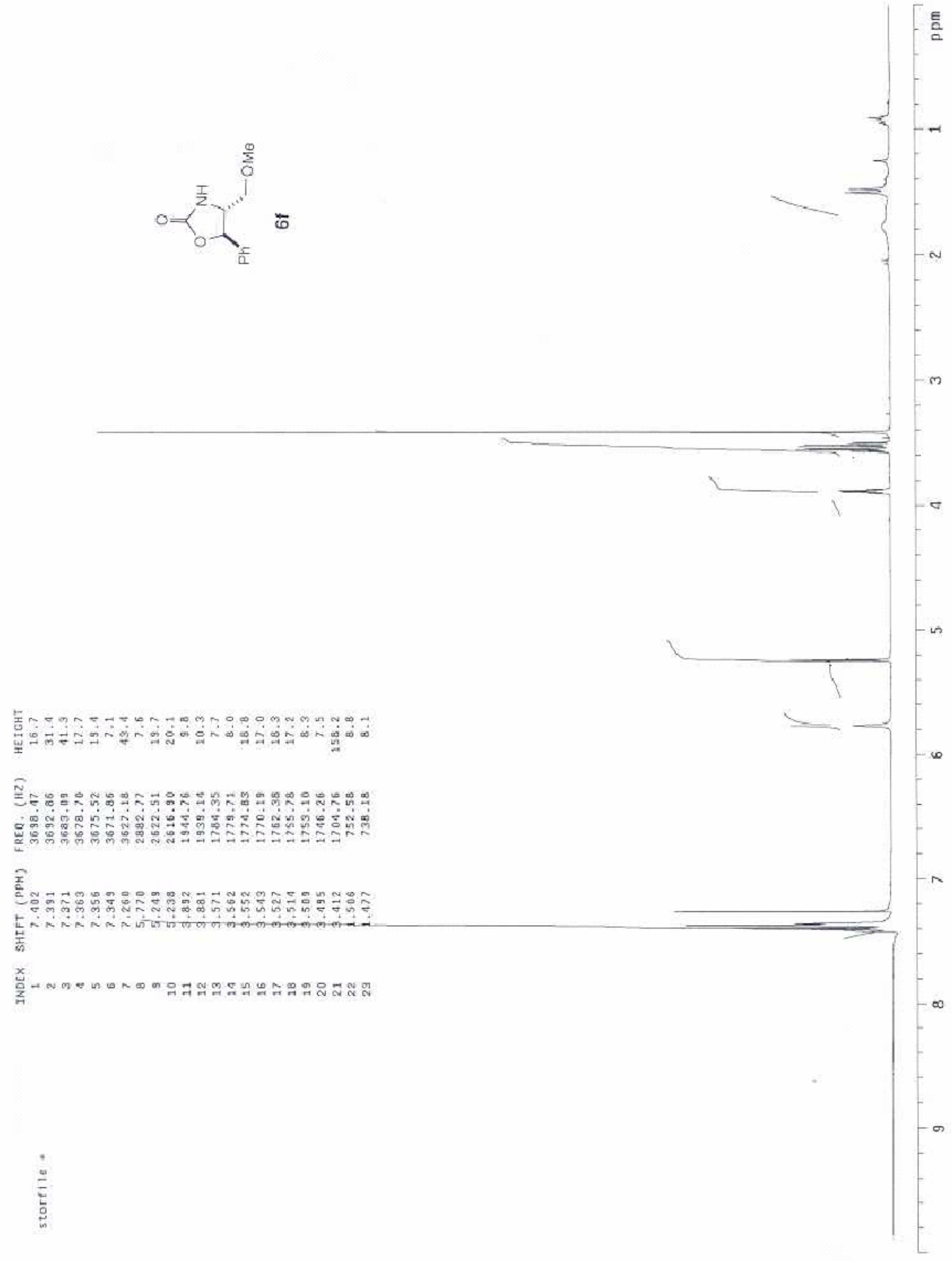




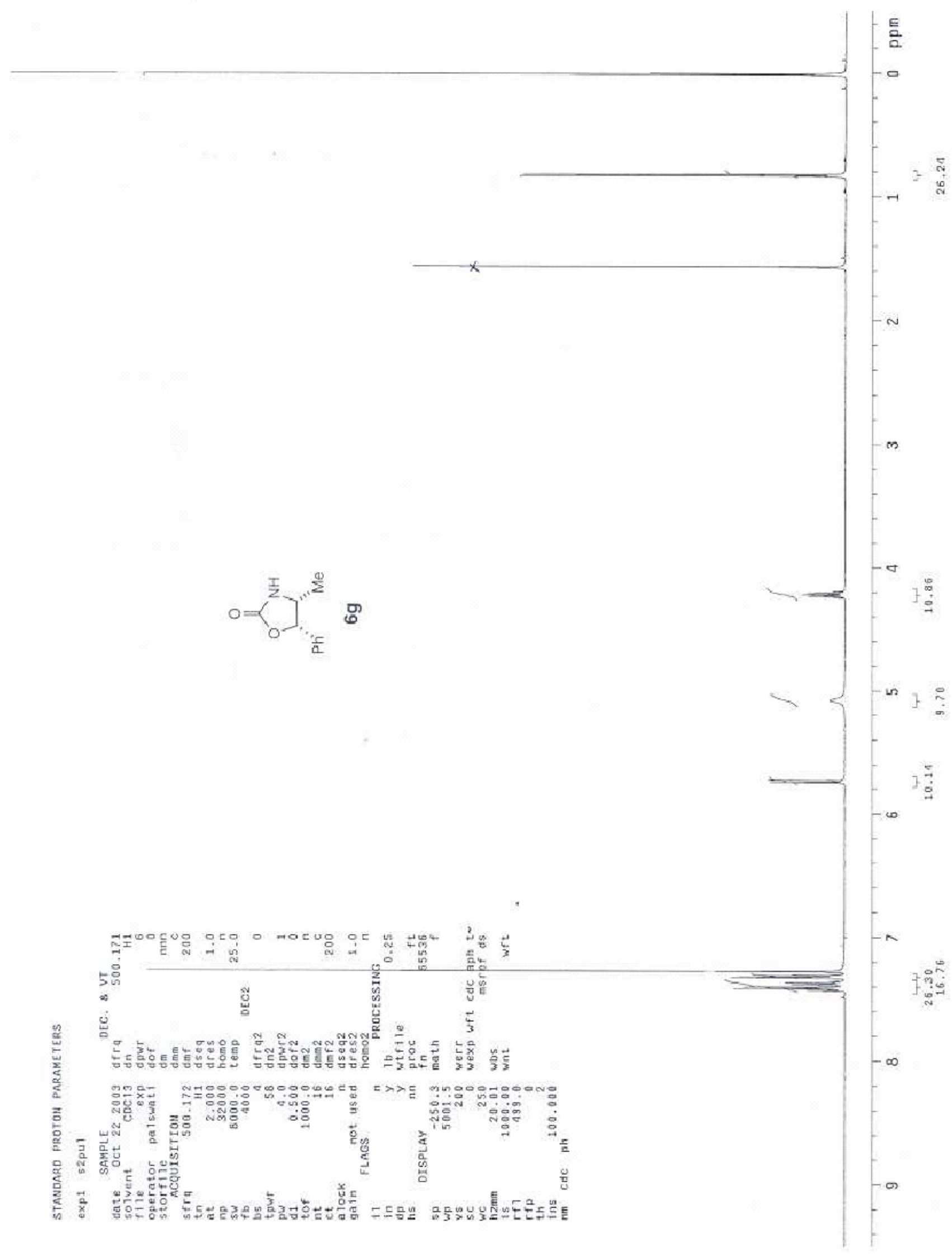




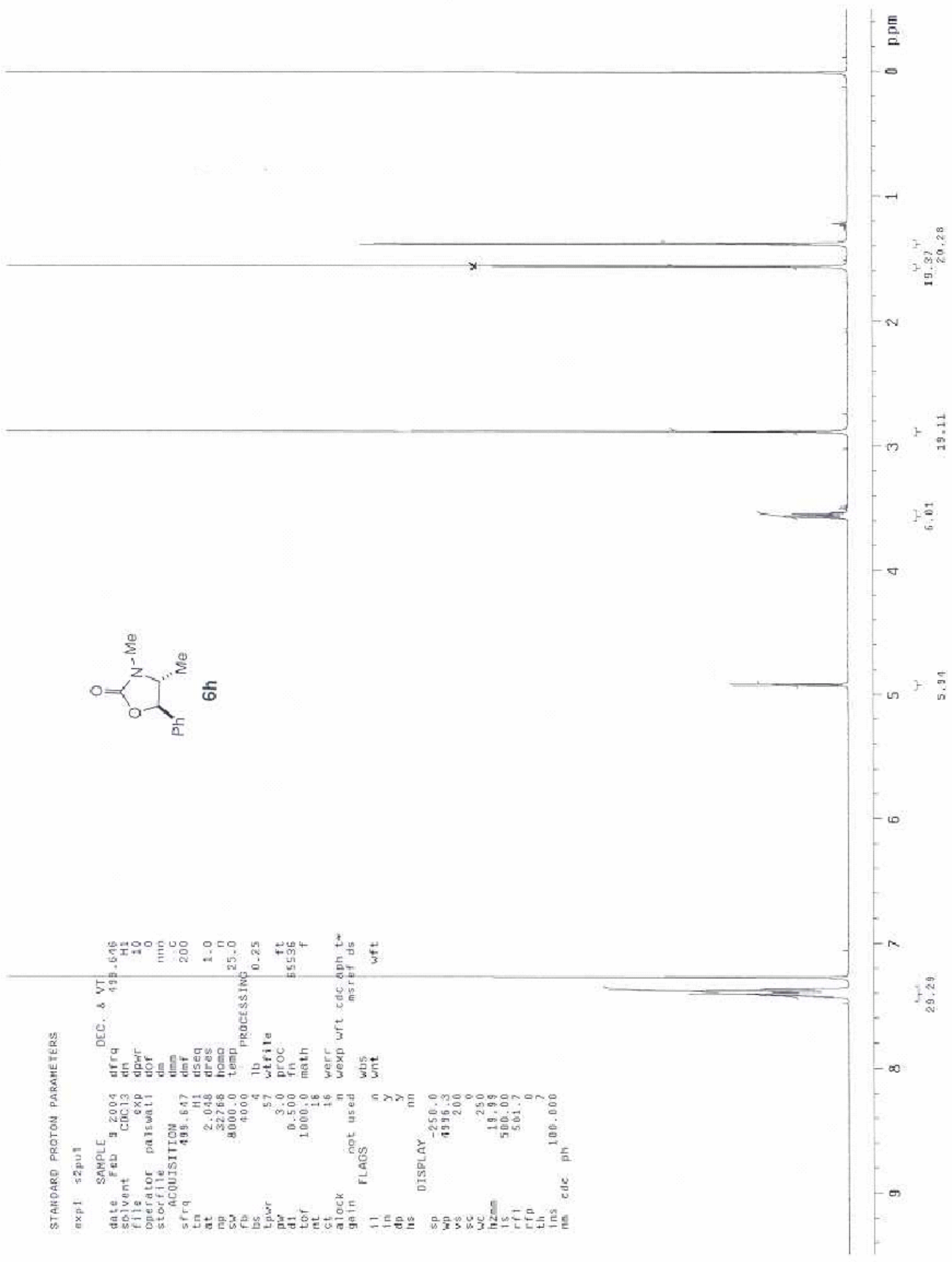




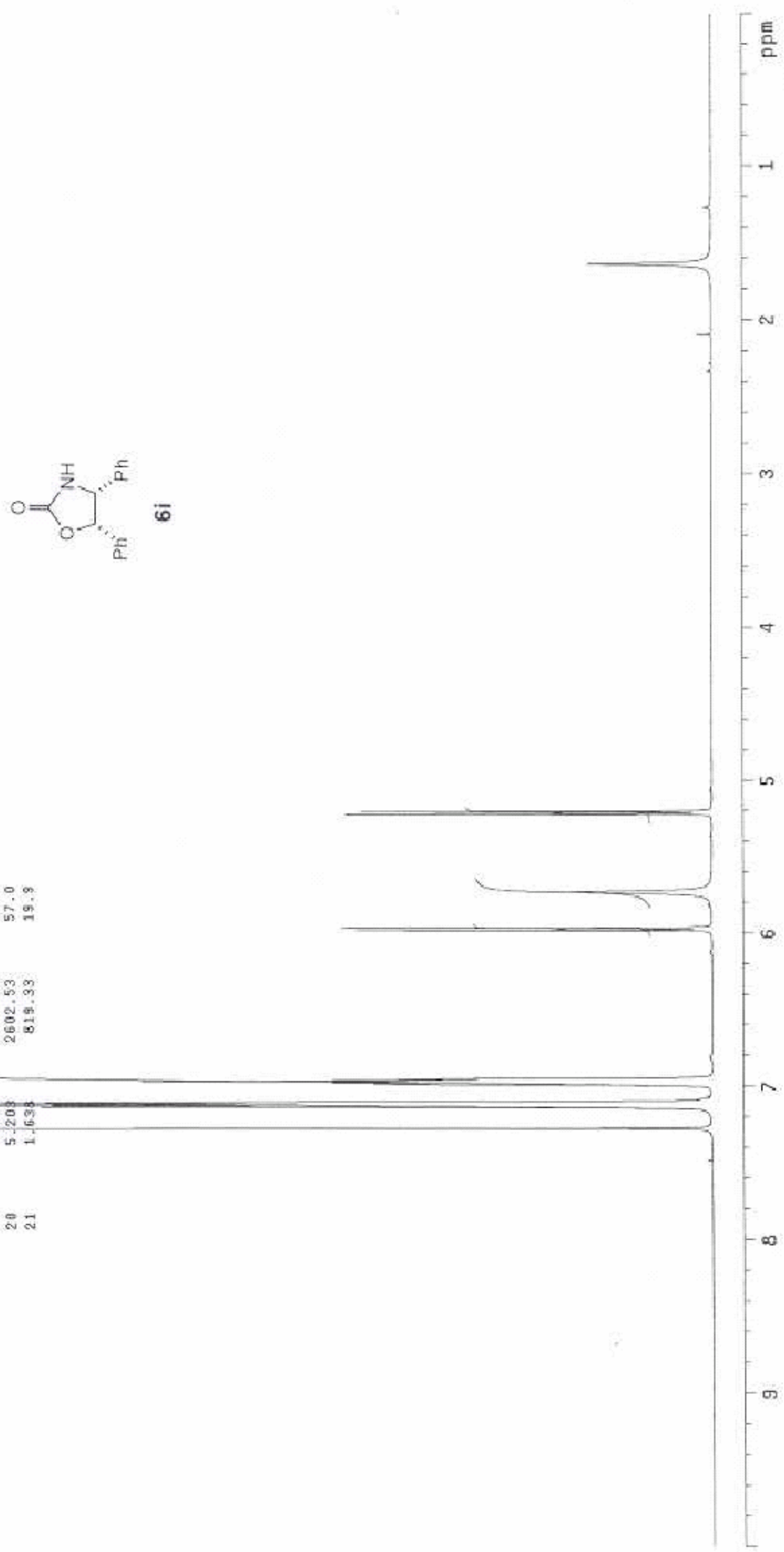

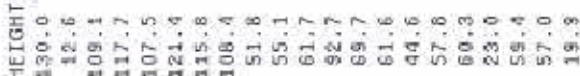

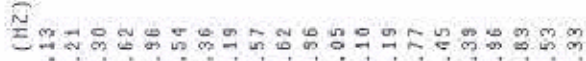

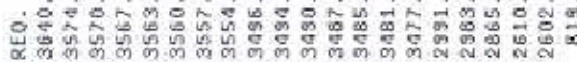

$\overline{\mathrm{E}}$

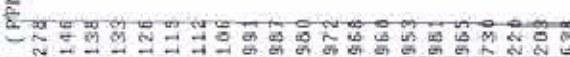

-77777 . $\frac{\mathrm{c}}{\frac{\mathrm{m}}{\mathrm{m}}}$

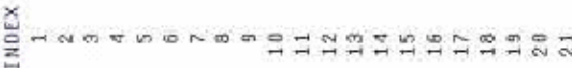

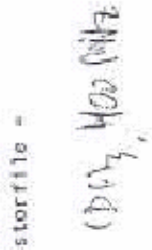




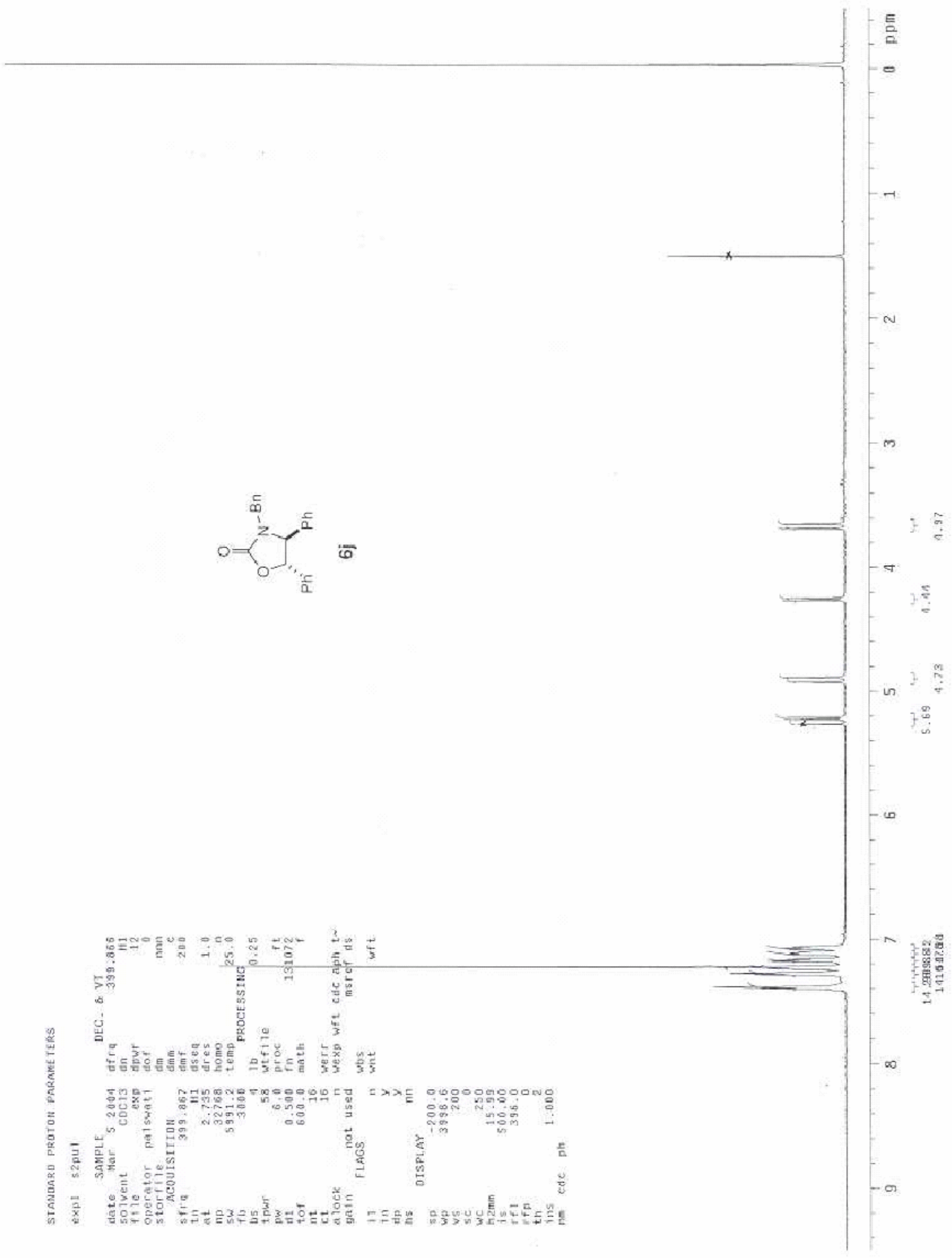




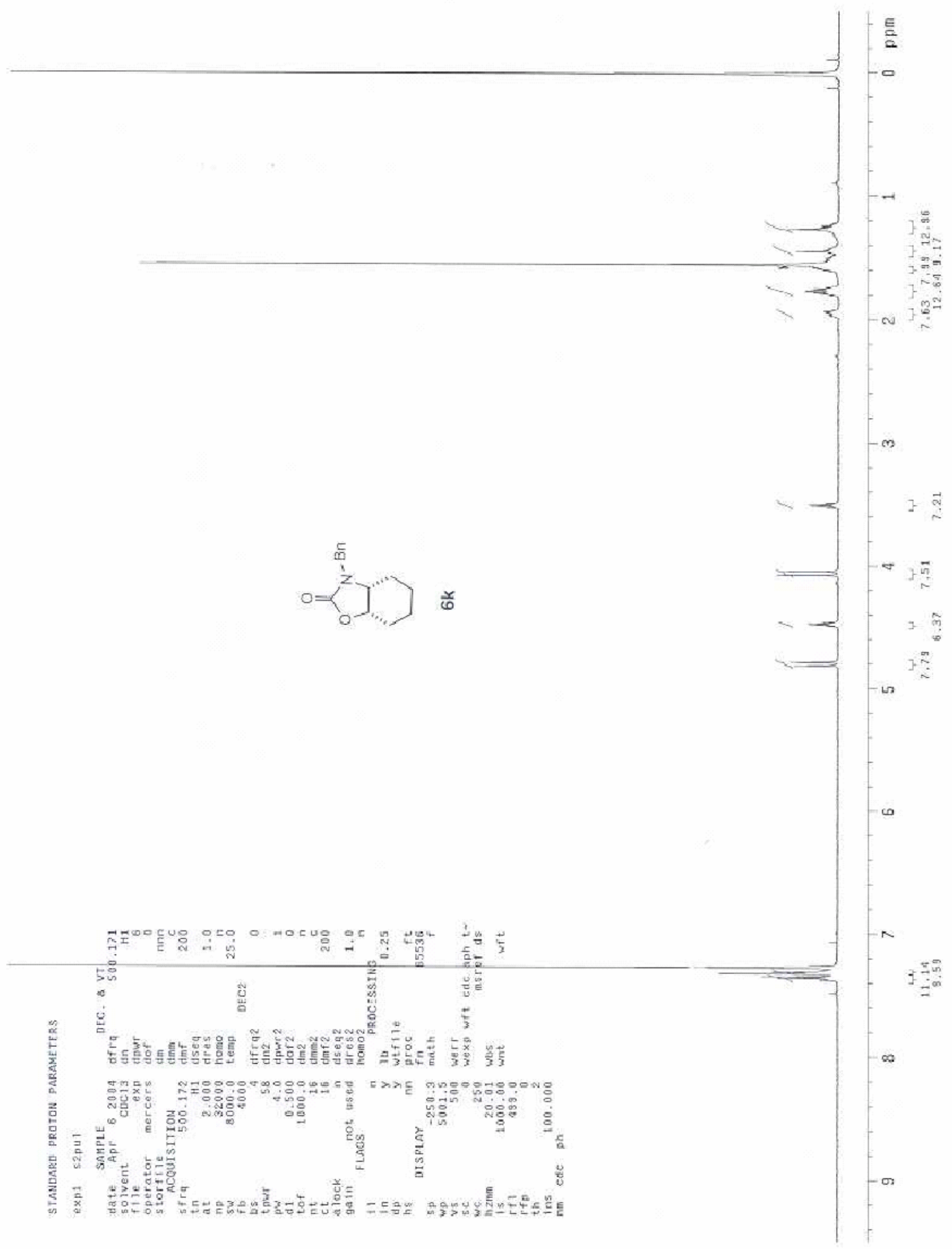




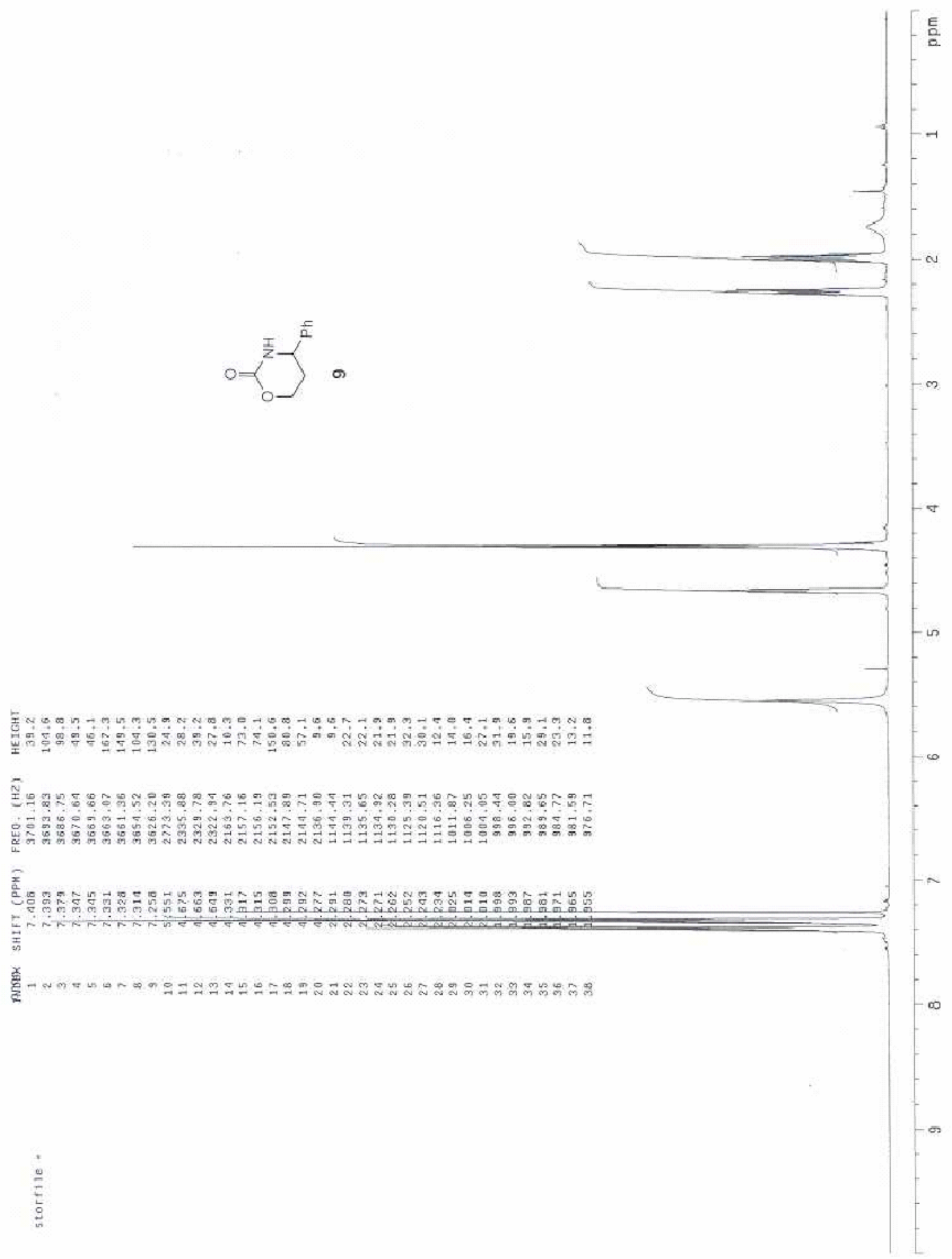




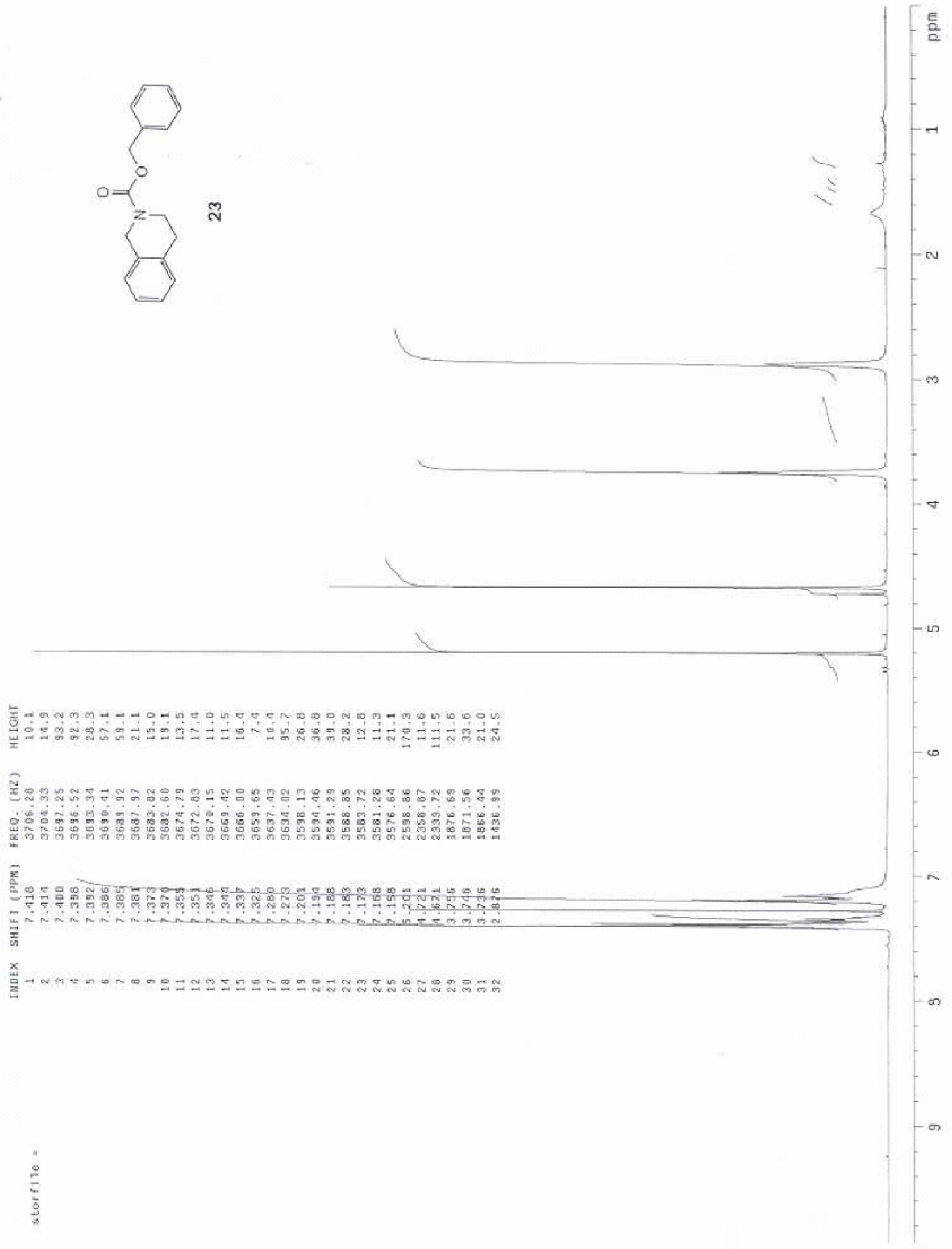


Copies of the LCMS and HRMS spectra from the carboxylation/Mitsunobu cyclization reaction of $4 \mathrm{~g}$ using ${ }^{18} \mathrm{O}$-labeled $\mathrm{CO}_{2}$ to give 10 (eq 2):

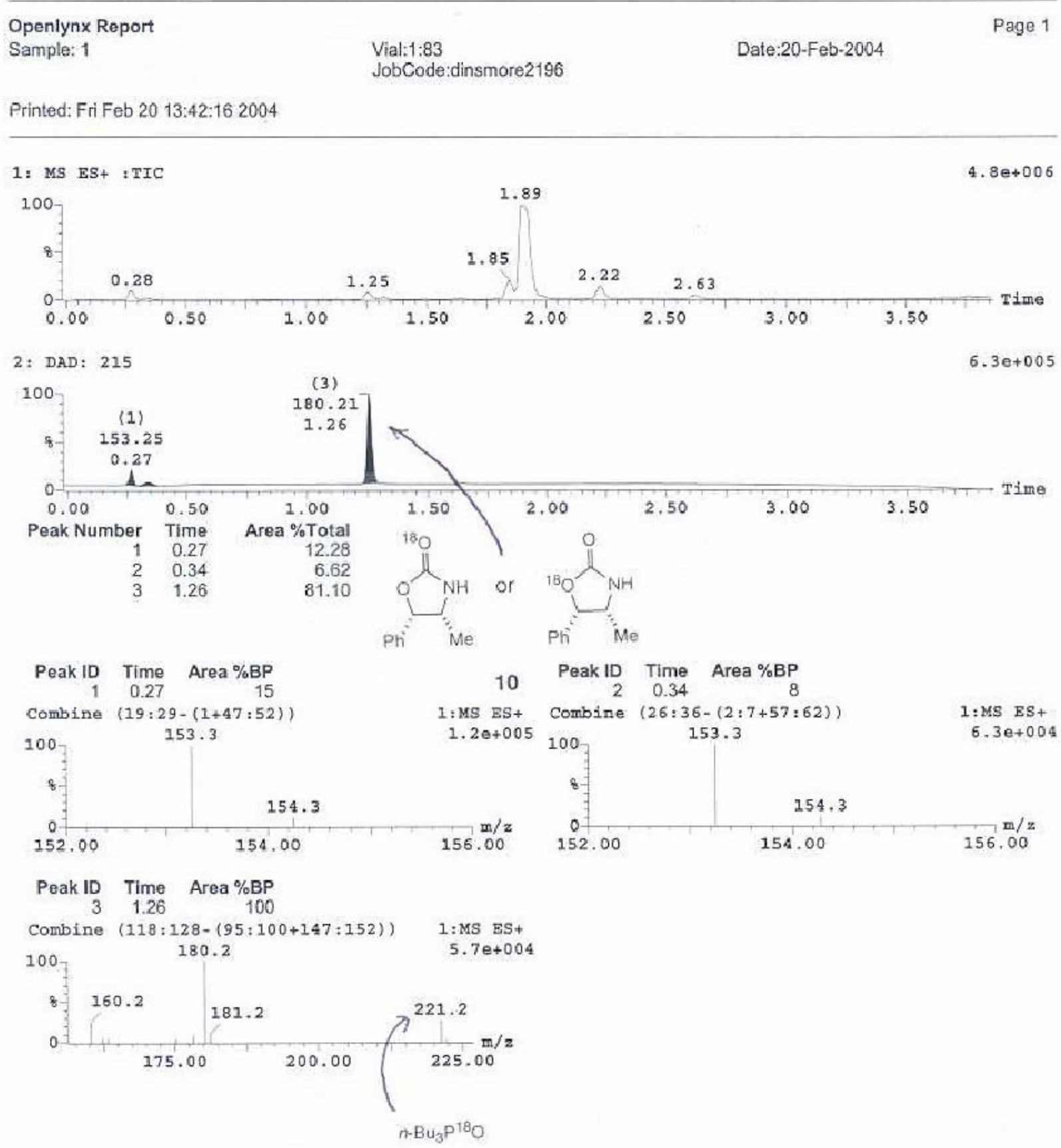




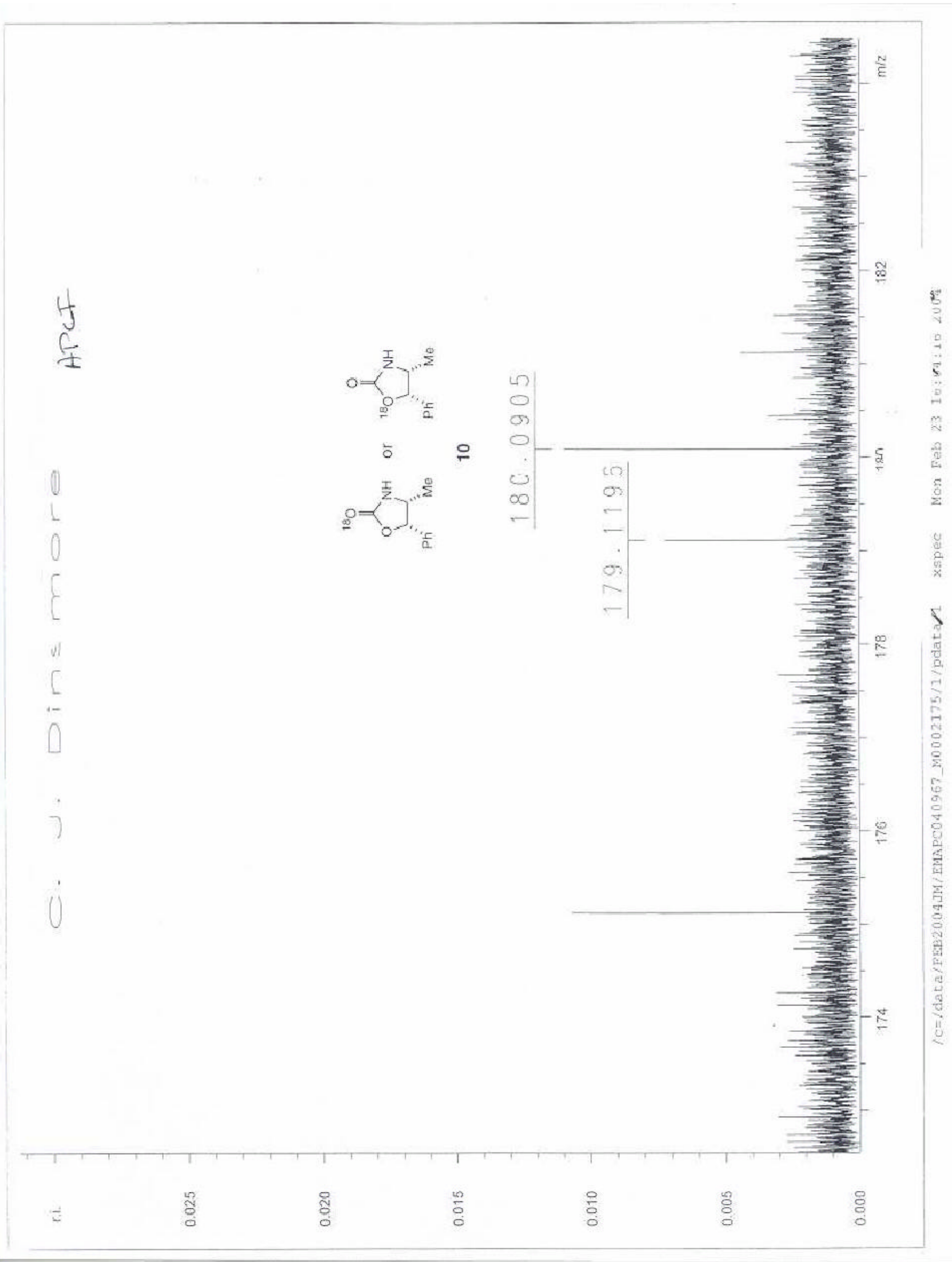


J

U

i

E

4)

f

-

Q
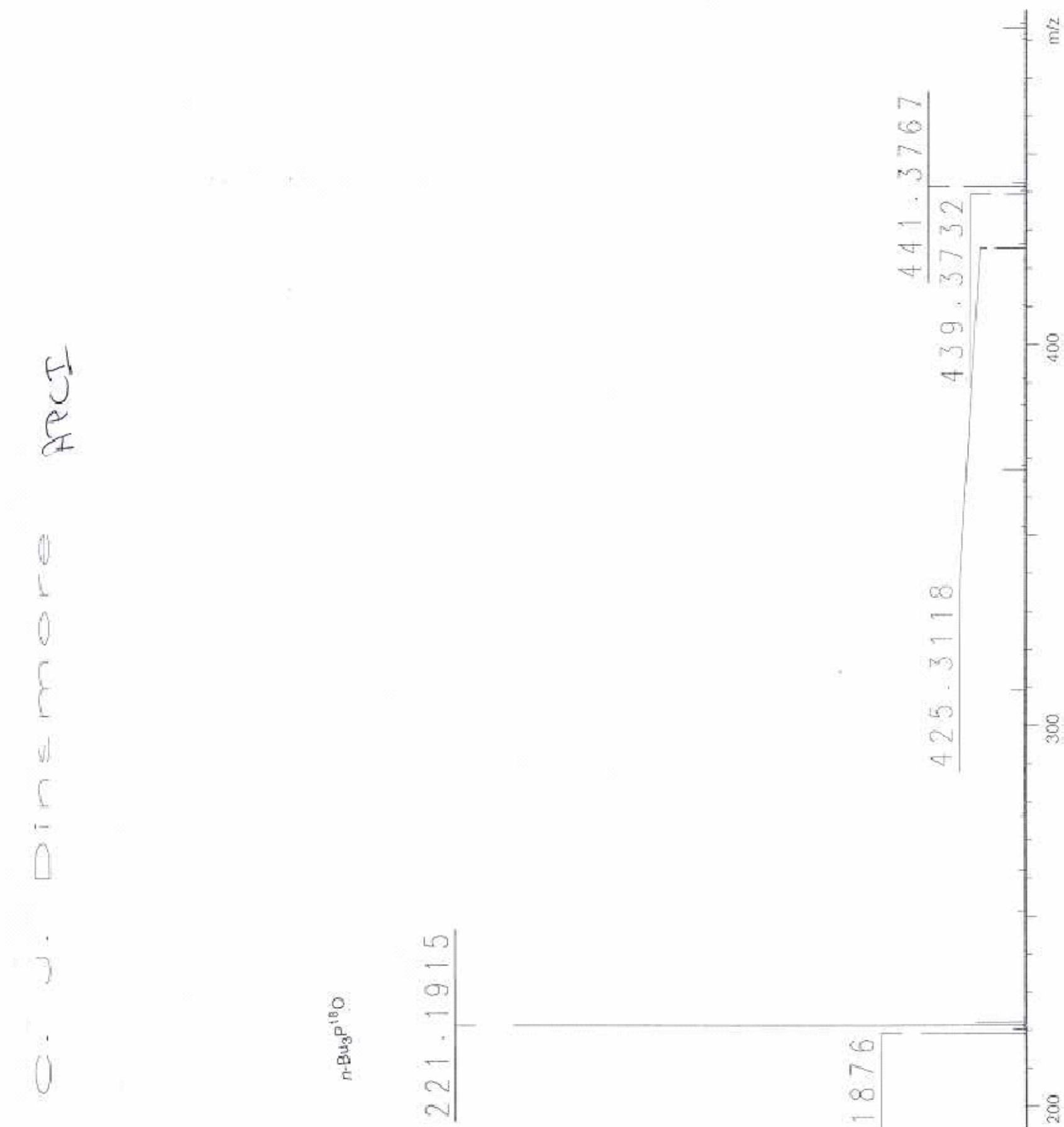

है
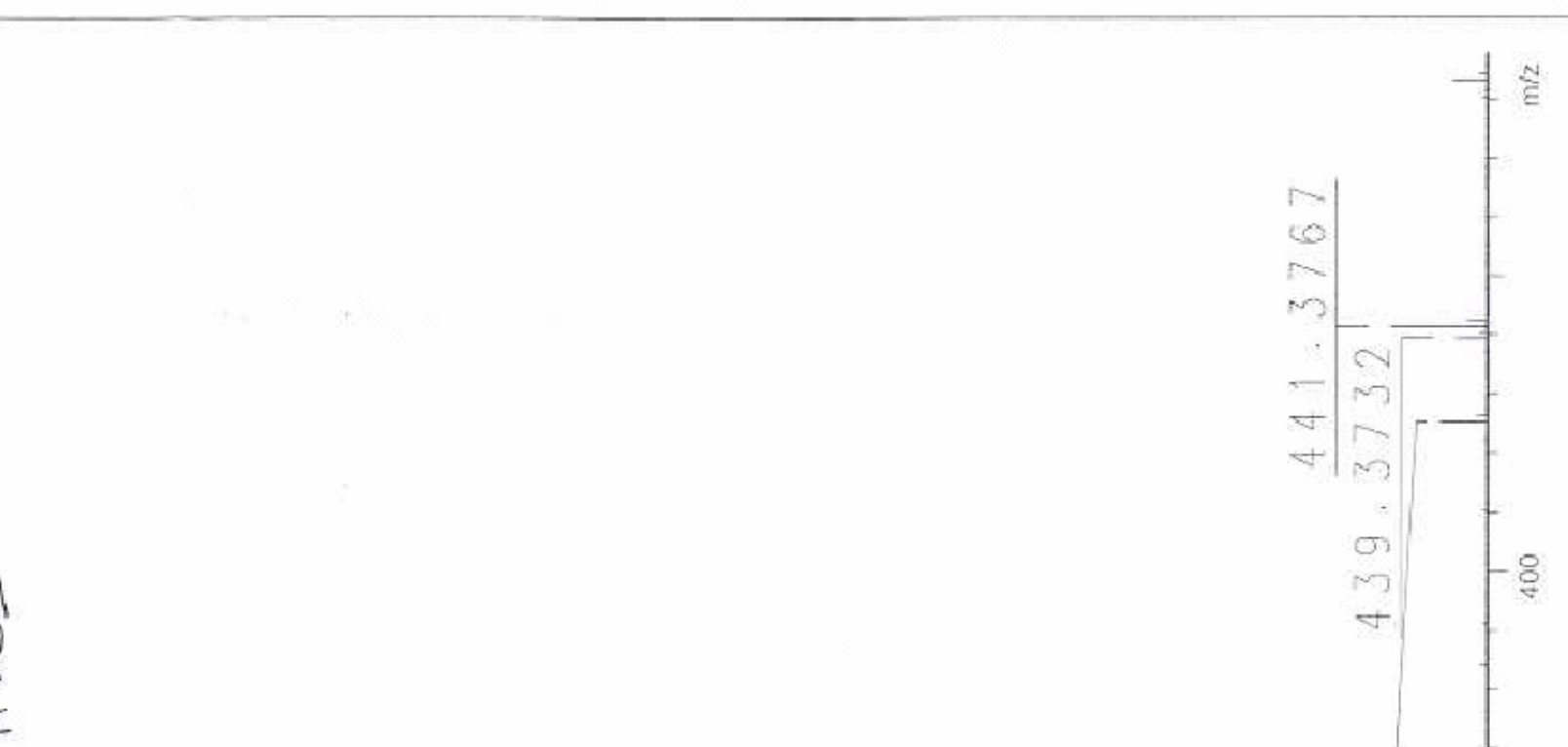

$$
+
$$

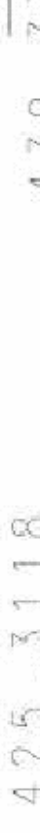

$\sigma$

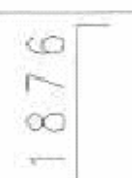

क.

ए

.
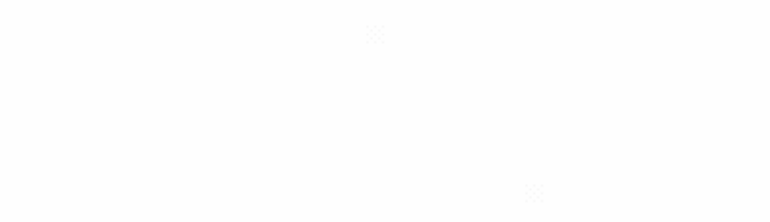
Copies of the LCMS and HRMS spectra from the carboxylation/Mitsunobu cyclization reaction of $4 \mathrm{~h}$ using ${ }^{18} \mathrm{O}$-labeled $\mathrm{CO}_{2}$ to give 11 (eq 3):

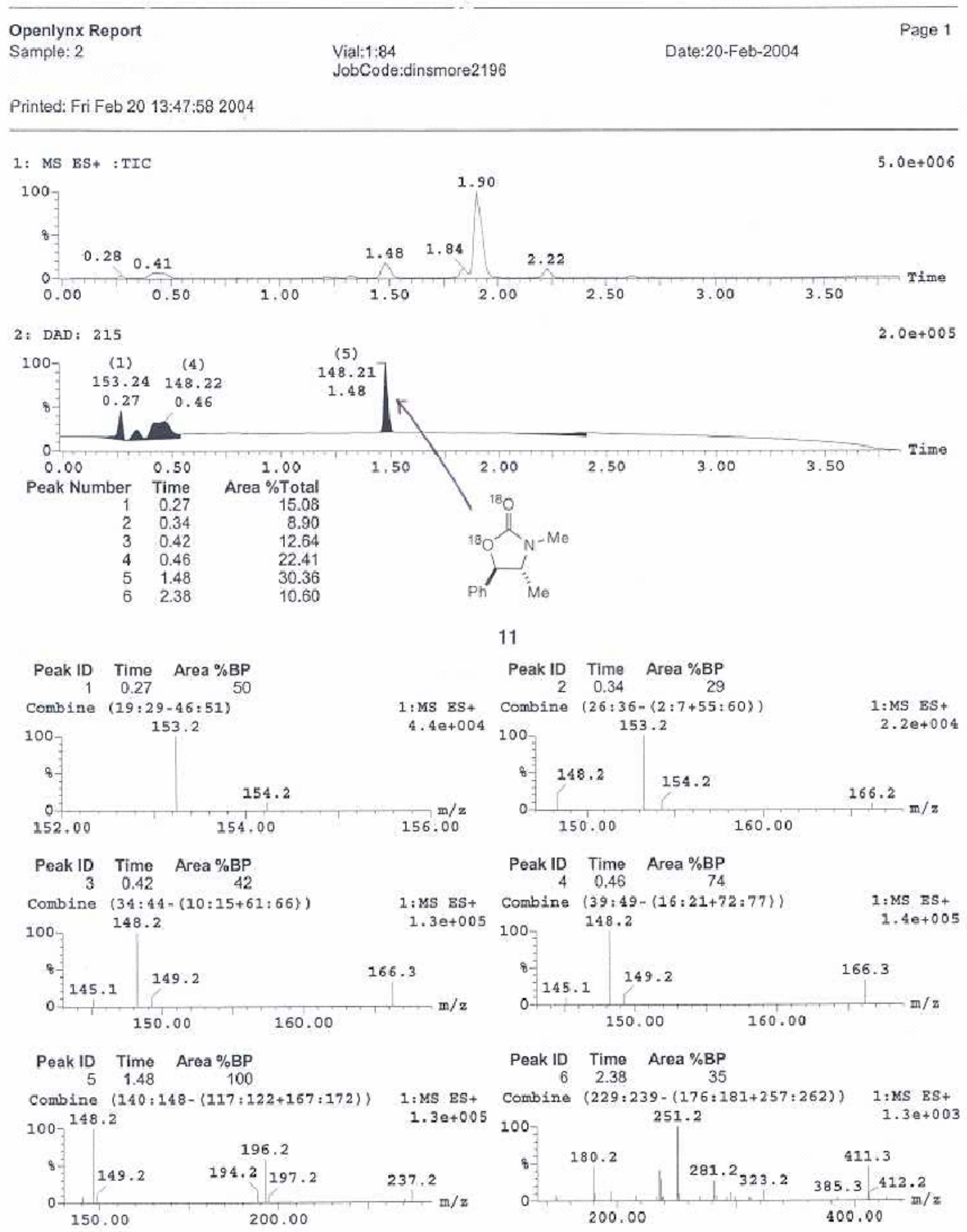




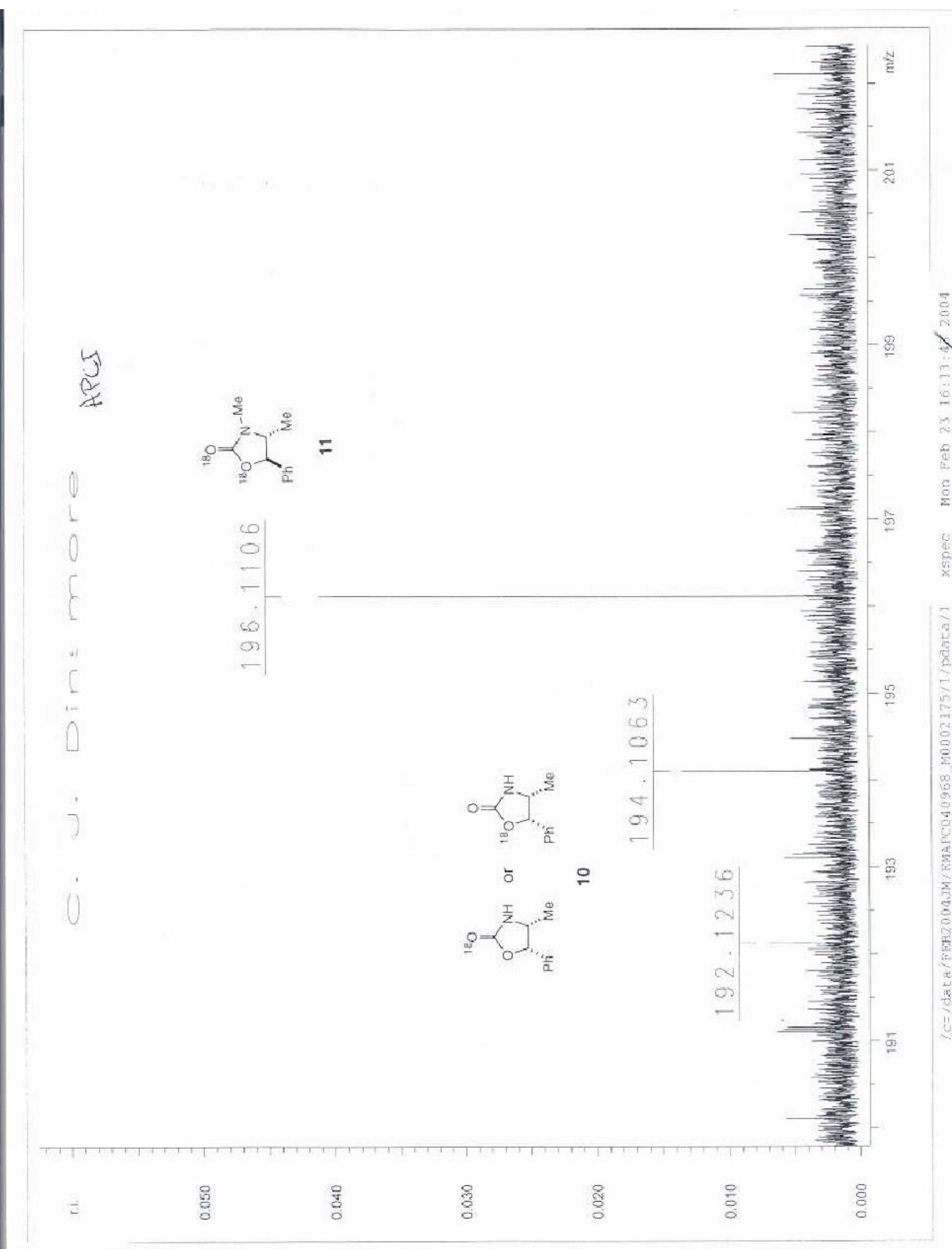




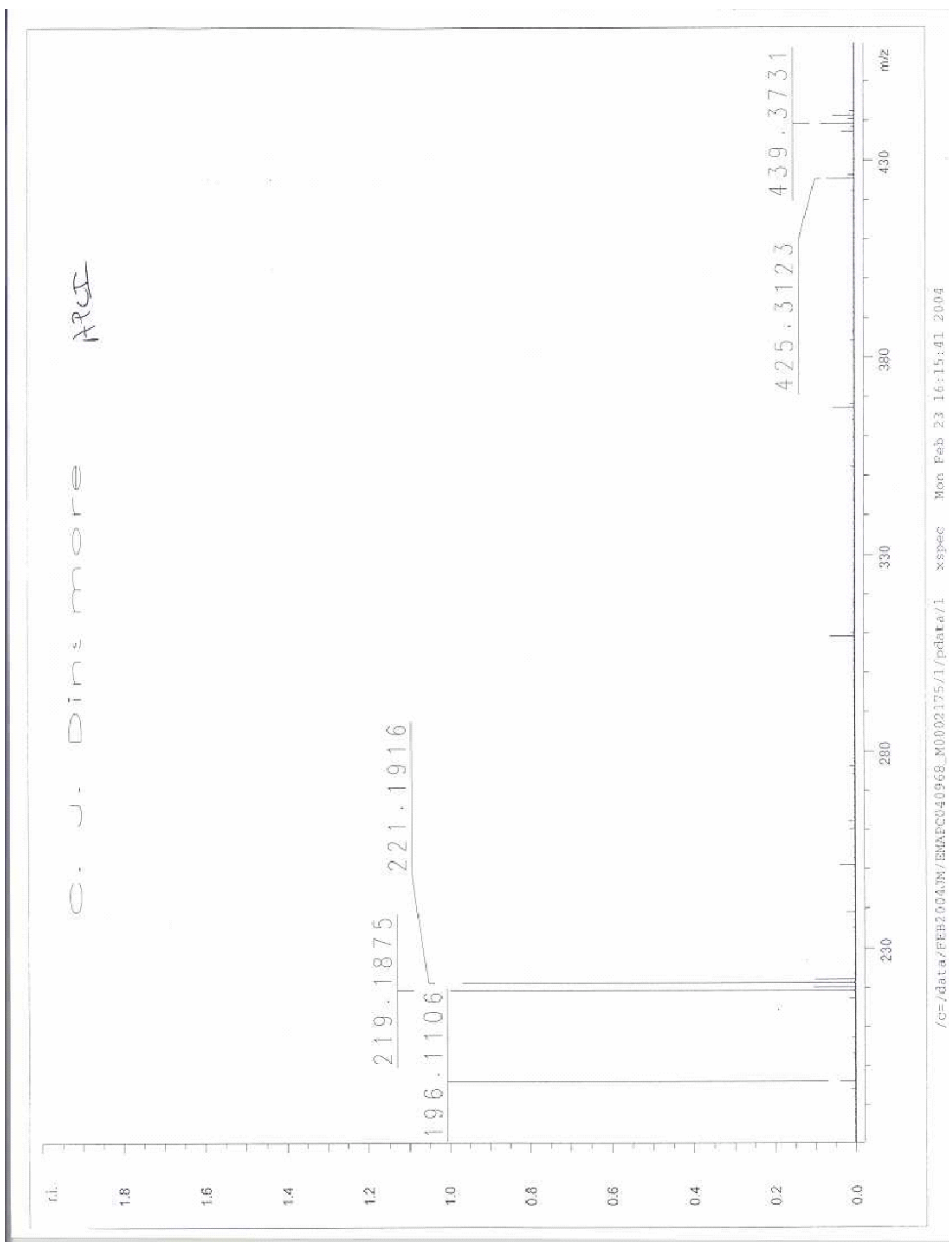

